\title{
A Conformal Energy-Conserved Method for Maxwell's Equations with Perfectly Matched Layers
}

\author{
Chaolong Jiang ${ }^{1}$, Jin Cui ${ }^{1,2}$ and Yushun Wang ${ }^{1, *}$ \\ 1 Jiangsu Provincial Key Laboratory for NSLSCS, School of Mathematical Sciences, \\ Nanjing Normal University, Nanjing 210023, China. \\ 2 Department of Basic Sciences, Nanjing College of Information and Technology, \\ Nanjing 210023, China.
}

Received 21 October 2017; Accepted (in revised version) 13 February 2018

\begin{abstract}
In this paper, a conformal energy-conserved scheme is proposed for solving the Maxwell's equations with the perfectly matched layer. The equations are split as a Hamiltonian system and a dissipative system, respectively. The Hamiltonian system is solved by an energy-conserved method and the dissipative system is integrated exactly. With the aid of the Strang splitting, a fully-discretized scheme is obtained. The resulting scheme can preserve the five discrete conformal energy conservation laws and the discrete conformal symplectic conservation law. Based on the energy method, an optimal error estimate of the scheme is established in discrete $L^{2}$-norm. Some numerical experiments are addressed to verify our theoretical analysis.
\end{abstract}

AMS subject classifications: 65M12, 65M15, 65M70

Key words: Maxwell's equations, Fourier pseudo-spectral method, error estimate, conformal conservation law, PML.

\section{Introduction}

Since the initial work of Yee [38], numerical methods have been widely used in solving electromagnetic problems. However, one of the inconveniences of the numerical methods lies in the fact that the Maxwell's equations have to be solved in a bounded domain. Thus, in order to absorb the outgoing waves, one needs to apply some special conditions on the boundaries of the computational domain. In Refs. [2,3], Bérenger firstly proposed the perfectly matched layer (PML) technique, which was based on the use of an absorbing layer especially designed to absorb the electromagnetic waves without reflection from the vacuum-layer interfaces. The basic idea of the PML technique of Bérenger was based on modifying the Maxwell's equations in the absorbing layer. The resulting equations

*Corresponding author. Email addresses: wangyushun@njnu.edu.cn (Y. Wang), chaolong-jiang@126.com (C. Jiang), 1667276479@qq. com (J. Cui) 
are commonly referred to as the Maxwell's equations with the PML $[19,20]$. Due to the simplicity, the versatility, and the robust treatment of corners in the practical applications, devising efficient numerical methods for the Maxwell's equations with the PML attracts a lot of interest.

It is well-known that structure-preserving methods or geometric numerical methods have exhibited significant superiority over traditional methods in solving Hamiltonian ordinary differential equations (ODEs) and Hamiltonian partial differential equations (PDEs) (e.g., see Refs. [5, 13, 17, 37] and references therein). In the past few decades, various structure-preserving schemes have been developed for the Maxwell's equations. In Refs. [8, 18, 23, 33, 36, 39], symplectic and multi-symplectic schemes of the Maxwell's equations in an isotropic, lossless and sourceless medium were proposed. In Ref. [11], Chen et al. proposed an energy-conserved splitting method for two dimensional (2D) Maxwell's equations in the isotropic, lossless and sourceless medium. Further analysis in three dimensional (3D) case was investigated in Ref. [12]. Other works on the energyconserved methods for the Maxwell's equations in the isotropic, lossless and sourceless medium can be found in Refs. $[6,7,22]$.

However, since the system of the Maxwell's equations with the PML is neither a conservative system nor a Hamiltonian system, the energy-conserved methods, which were developed for the Maxwell's equations, will lose their advantages when applied directly to the Maxwell's equations with the PML. Thus, designing the numerical schemes for the Maxwell's equations with the PML is challenging. In Ref. [20], by virtue of the splitting technique, Hong, Ji and Kong proposed an energy-dissipation splitting finitedifference time-domain (FDTD) method for the 2D Maxwell's equations with the PML. Subsequently, Hong and Ji [19] studied the energy evolution of multi-symplectic methods for the 3D case. In Ref. [32], Birkhoffian multi-symplectic methods for the Maxwell's equations with the PML were investigated by Su and Li. However, most of existing methods have low order accuracy in space and the rigorous error estimate is not established well.

Recently, there has been growing interest in conformal methods for Hamiltonian systems with a linear damping term (e.g., see Refs. [14, 27, 29]). The conformal method provided clear advantages in preserving the conformal conservation laws and long time simulations over standard methods $[4,29]$. Other works most related to the conformal method can be found in Refs. [24,34,35]. However, there has been no reference considering a conformal Fourier pseudo-spectral scheme for the Maxwell's equations with the PML to the best of our knowledge. It is shown that Fourier pseudo-spectral methods with a high order accuracy have exhibited obvious superiority over the conventional finite difference method in simulating electromagnetic waves [25] and played an important role in keeping the physical properties of primitive problems (e.g., see Refs. [10,15,30,37] and references therein). Thus, our main attention is focusing on the following two aspects:

1. We propose a novel conformal Fourier pseudo-spectral scheme (CFPS) for the Maxwell's equations with the PML. We show that the proposed scheme can pre- 
serve the five conformal energy conservation laws and the conformal symplectic conservation law of the Maxwell's equations with the PML.

2. An optimal error estimate of the proposed scheme is established, which shows that the scheme is of second order accuracy in time and spectral accuracy in space in discrete $L^{2}$-norm.

The outline of this paper is organized as follows. In Section 2, a conformal Hamiltonian system of the Maxwell's equations with the PML is introduced and a conformal energy-conserved scheme is proposed. In Section 3, we first show that the proposed scheme can preserve the five discrete conformal conservation laws and the discrete symplectic conservation law. Then, based on the energy method, an optimal error estimate of the scheme is established. Some numerical experiments are presented in Section 4. We draw some conclusions in Section 5.

\section{A conformal Fourier pseudo-spectral scheme}

This section has two goals. Firstly, the conformal Hamiltonian system of the Maxwell's equations with the PML is introduced. Secondly, the derivation of the novel conformal energy-conserved scheme is expounded.

\subsection{Conformal Hamiltonian system}

The Maxwell's equations in the isotropic, lossless and sourceless medium can be described, as follows:

$$
\left\{\begin{array}{l}
-\frac{\partial E}{\partial t}+\frac{1}{\epsilon} \nabla \times \boldsymbol{H}=0 \\
\frac{\partial \boldsymbol{H}}{\partial t}+\frac{1}{\mu} \nabla \times \boldsymbol{E}=0
\end{array}\right.
$$

with initial conditions

$$
\boldsymbol{E}(x, y, z, 0)=\boldsymbol{E}_{0}(x, y, z), \quad \boldsymbol{H}(x, y, z, 0)=\boldsymbol{H}_{0}(x, y, z), \quad(x, y, z) \in \Omega \subset \mathbb{R}^{3},
$$

where $\boldsymbol{E}=\left(E_{x}, E_{y}, E_{z}\right)^{T}$ is the electric field intensity, $\boldsymbol{H}=\left(H_{x}, H_{y}, H_{z}\right)^{T}$ is the magnetic field intensity, constant scalars $\mu$ and $\epsilon$ are the magnetic permeability and the electric permittivity, respectively. In this paper, we consider cuboid domain $\Omega=\left[x_{L}, x_{R}\right] \times\left[y_{L}, y_{R}\right] \times$ $\left[z_{L}, z_{R}\right]$ and impose periodic boundary conditions on the boundary $\partial \Omega \times[0, T]$.

In general, the perfect electric conducting boundary condition is imposed on $\partial \Omega \times$ $[0, T]$ of $(2.1)$. However, with the aid of the Bérenger's PML technique [2,3], the periodic boundary condition is valid in practical computations. For more details, please refer to Ref. [25]. 
According to Bérenger [3], in the PML medium, each component of the electromagnetic field is split into two parts. Then, the six components of (2.1) yield 12 subcomponents denoted as $E_{x y}, E_{x z}, E_{y z}, E_{y x}, E_{z x}, E_{z y}, H_{x y}, H_{x z}, H_{y z}, H_{y x}, H_{z x}, H_{z y}$, and the Maxwell's equations are replaced by 12 equations,

$$
\begin{aligned}
& \epsilon \frac{\partial E_{x y}}{\partial t}+\sigma_{y} E_{x y}=\frac{\partial\left(H_{z x}+H_{z y}\right)}{\partial y}, \quad \epsilon \frac{\partial E_{x z}}{\partial t}+\sigma_{z} E_{x z}=-\frac{\partial\left(H_{y z}+H_{y x}\right)}{\partial z}, \\
& \epsilon \frac{\partial E_{y z}}{\partial t}+\sigma_{z} E_{y z}=\frac{\partial\left(H_{x y}+H_{x z}\right)}{\partial z}, \quad \epsilon \frac{\partial E_{y x}}{\partial t}+\sigma_{x} E_{y x}=-\frac{\partial\left(H_{z x}+H_{z y}\right)}{\partial x}, \\
& \epsilon \frac{\partial E_{z x}}{\partial t}+\sigma_{x} E_{z x}=\frac{\partial\left(H_{y z}+H_{y x}\right)}{\partial x}, \quad \epsilon \frac{\partial E_{z y}}{\partial t}+\sigma_{y} E_{z y}=-\frac{\partial\left(H_{x y}+H_{x z}\right)}{\partial y}, \\
& \mu \frac{\partial H_{x y}}{\partial t}+\sigma_{y}^{*} H_{x y}=-\frac{\partial\left(E_{z x}+E_{z y}\right)}{\partial y}, \quad \mu \frac{\partial H_{x z}}{\partial t}+\sigma_{z}^{*} H_{x z}=\frac{\partial\left(E_{y z}+E_{y x}\right)}{\partial z}, \\
& \mu \frac{\partial H_{y z}}{\partial t}+\sigma_{z}^{*} H_{y z}=-\frac{\partial\left(E_{x y}+E_{x z}\right)}{\partial z}, \quad \mu \frac{\partial H_{y x}}{\partial t}+\sigma_{x}^{*} H_{y x}=\frac{\partial\left(E_{z x}+E_{z y}\right)}{\partial x}, \\
& \mu \frac{\partial H_{z x}}{\partial t}+\sigma_{x}^{*} H_{z x}=-\frac{\partial\left(E_{y z}+E_{y x}\right)}{\partial x}, \quad \mu \frac{\partial H_{z y}}{\partial t}+\sigma_{y}^{*} H_{z y}=\frac{\partial\left(E_{x y}+E_{x z}\right)}{\partial y},
\end{aligned}
$$

where the parameters $\left(\sigma_{x}, \sigma_{y}, \sigma_{z}, \sigma_{x}^{*}, \sigma_{y}^{*}, \sigma_{z}^{*}\right)$ are homogeneous to electric and magnetic conductivities.

If both $\sigma_{x}=\sigma_{y}=\sigma_{z}$ and $\sigma_{x}^{*}=\sigma_{y}^{*}=\sigma_{z}^{*}=0$, we can note that (2.2) yield the classical Maxwell's equations (2.1). Thus, the absorbing medium defined by (2.2) holds as particular cases of all usual media (vacuum, conductive media). If both $\frac{\sigma_{x}}{\epsilon}=\frac{\sigma_{y}}{\epsilon}=\frac{\sigma_{z}}{\epsilon}$ and $\frac{\sigma_{x}^{*}}{\mu}=\frac{\sigma_{y}^{*}}{\mu}=\frac{\sigma_{z}^{*}}{\mu}=\bar{\sigma},(2.2)$ reduces to

$$
\left\{\begin{array}{l}
-\frac{\partial \boldsymbol{E}}{\partial t}+\frac{1}{\epsilon} \nabla \times \boldsymbol{H}-\bar{\sigma} \boldsymbol{E}=0, \\
\frac{\partial \boldsymbol{H}}{\partial t}+\frac{1}{\mu} \nabla \times \boldsymbol{E}+\bar{\sigma} \boldsymbol{H}=0 .
\end{array}\right.
$$

Eqs. (2.3) can be rewritten as a conformal Hamiltonian system [4,27]

$$
J \partial_{t} z=\frac{\delta \mathcal{H}(z)}{\delta z}-\bar{\sigma} J z
$$

where $\boldsymbol{z}=\left[\boldsymbol{H}^{T}, \boldsymbol{E}^{T}\right]^{T}$,

$$
J=\left[\begin{array}{cc}
0 & \boldsymbol{I}_{3 \times 3} \\
-\mathbf{I}_{3 \times 3} & 0
\end{array}\right]
$$

and

$$
\mathcal{H}(\boldsymbol{z})=\int_{\Omega}\left[\frac{1}{2 \epsilon} \boldsymbol{H}^{T} \nabla \times \boldsymbol{H}+\frac{1}{2 \mu} \boldsymbol{E}^{T} \nabla \times \boldsymbol{E}\right] d x d y d z
$$


is the helicity Hamiltonian function [1].

The merit of the conformal Hamiltonian system (2.4) is that it possesses the following conformal Hamiltonian energy conservation law

$$
\frac{d}{d t} \mathcal{H}=-2 \bar{\sigma} \mathcal{H}, \quad \mathcal{H}=\int_{\Omega}\left[\frac{1}{2 \epsilon} \boldsymbol{H}^{T} \nabla \times \boldsymbol{H}+\frac{1}{2 \mu} \boldsymbol{E}^{T} \nabla \times \boldsymbol{E}\right] d x d y d z,
$$

and the conformal symplectic conservation law

$$
\frac{d}{d t} \omega=-2 \bar{\sigma} \omega, \quad \omega=\int_{\Omega}\left[\delta E_{x} \wedge \delta H_{x}+\delta E_{y} \wedge \delta H_{y}+\delta E_{z} \wedge \delta H_{z}\right]
$$

where $\wedge$ is the wedge product.

Remark 2.1. It should be remarked that (2.4) is not valid for the $2 \mathrm{D}$ transverse-electric (TE) waves in the PML medium, which will be investigated in Section 4.

In addition, (2.3) also admits the following conformal energy conservation laws.

Lemma 2.1. [32] Let $\boldsymbol{H}^{n}$ and $\boldsymbol{E}^{n}$ be the solutions of (2.3) and satisfy the periodic boundary condition, then the following conformal energy conservation laws hold

$$
\begin{array}{ll}
\frac{d}{d t} \mathcal{H}_{1}=-2 \bar{\sigma} \mathcal{H}_{1}, & \mathcal{H}_{1}=\int_{\Omega}\left[\frac{\mu}{2} \boldsymbol{H}^{T} \boldsymbol{H}+\frac{\epsilon}{2} \boldsymbol{E}^{T} \boldsymbol{E}\right] d x d y d z, \\
\frac{d}{d t} \mathcal{H}_{2}=-2 \bar{\sigma} \mathcal{H}_{2}, & \mathcal{H}_{2}=\int_{\Omega}\left[\frac{\epsilon}{2} \partial_{t} \boldsymbol{E}^{T} \partial_{t} \boldsymbol{E}+\frac{\mu}{2} \partial_{t} \boldsymbol{H}^{T} \partial_{t} \boldsymbol{H}\right] d x d y d z, \\
\frac{d}{d t} \mathcal{H}_{3}=-2 \bar{\sigma} \mathcal{H}_{3}, & \mathcal{H}_{3}=\int_{\Omega}\left[\epsilon \partial_{w} \boldsymbol{E}^{T} \partial_{w} \boldsymbol{E}+\mu \partial_{w} \boldsymbol{H}^{T} \partial_{w} \boldsymbol{H}\right] d x d y d z, \\
\frac{d}{d t} \mathcal{H}_{4}=-2 \bar{\sigma} \mathcal{H}_{4}, & \mathcal{H}_{4}=\int_{\Omega}\left[\epsilon \partial_{t w} \boldsymbol{E}^{T} \partial_{t w} \boldsymbol{E}+\mu \partial_{t w} \boldsymbol{H}^{T} \partial_{t w} \boldsymbol{H}\right] d x d y d z,
\end{array}
$$

where $w=x, y$, or $z$, and hereafter.

Remark 2.2. It is remarked that (2.7) shows that the electromagnetic energy in the Poynting's theorem [21] dissipates exponentially along time for the Maxwell's equations with the PML.

Remark 2.3. As pointed out in Ref. [32], (2.3) is perhaps not always met in practical computations, but it is the key condition of the PML for solving unbounded electromagnetic problems. Therefore, the investigation of numerical methods for the Maxwell's equations in such special case is also very important.

\subsection{Fourier pseudo-spectral approximation of the spatial derivatives}

In this subsection, the Maxwell's equations with the PML are approximated by the Fourier pseudo-spectral method in space. Let $\Omega_{h}=\left\{\left(x_{j}, y_{k}, z_{m}\right) \mid x_{j}=x_{L}+(j-1) h_{x}, y_{k}=y_{L}+(k-\right.$ 
1) $\left.h_{y}, z_{m}=z_{L}+(m-1) h_{z} ; j=1, \cdots, N_{x}, k=1, \cdots, N_{y}, m=1, \cdots, N_{z}\right\}$ be a partition of $\Omega$ with the mesh size $h_{w}=\frac{L_{w}}{N_{w}}$, where $L_{w}=w_{R}-w_{L}$ and $N_{w}$ is an even integers. Let $\Omega_{\tau}=\left\{t_{n} \mid t_{n}=\right.$ $n \tau ; 0 \leqslant n \leqslant M\}$ be a uniform partition of $[0, T]$ with the time step $\tau=\frac{T}{M}$ and $\Omega_{h \tau}=\Omega_{h} \times \Omega_{\tau}$.

We define

$$
S_{N}^{\prime \prime \prime}=\operatorname{span}\left\{g_{j}(x) g_{k}(y) g_{m}(z), j=1, \cdots, N_{x}, k=1, \cdots, N_{y}, m=1, \cdots, N_{z}\right\},
$$

as the interpolation space, where $g_{N_{x}}(x), g_{N_{y}}(y)$ and $g_{N_{z}}(z)$ are trigonometric polynomials of degree $N_{x} / 2, N_{y} / 2$ and $N_{z} / 2$, given respectively by

$$
\begin{aligned}
& g_{j}(x)=\frac{1}{N_{x}} \sum_{l=-N_{x} / 2}^{N_{x} / 2} \frac{1}{a_{l}} e^{\mathrm{i} l \mu_{x}\left(x-x_{j}\right)}, \quad g_{k}(y)=\frac{1}{N_{y}} \sum_{p=-N_{y} / 2}^{N_{y} / 2} \frac{1}{b_{p}} e^{\mathrm{i} p \mu_{y}\left(y-y_{k}\right)}, \\
& g_{m}(z)=\frac{1}{N_{z}} \sum_{q=-N_{z} / 2}^{N_{z} / 2} \frac{1}{c_{q}} e^{\mathrm{i} q \mu_{z}\left(z-z_{m}\right)},
\end{aligned}
$$

with

$$
a_{l}=\left\{\begin{array}{ll}
1, & |l|<\frac{N_{x}}{2}, \\
2, & |l|=\frac{N_{x}}{2},
\end{array}, \quad b_{p}=\left\{\begin{array}{ll}
1, & |p|<\frac{N_{y}}{2}, \\
2, & |p|=\frac{N_{y}}{2},
\end{array}, \quad c_{q}= \begin{cases}1, & |q|<\frac{N_{z}}{2}, \\
2, & |q|=\frac{N_{z}}{2},\end{cases}\right.\right.
$$

and $\mu_{w}=\frac{2 \pi}{L_{w}}$.

We define the interpolation operator $I_{N}: C(\Omega) \rightarrow S_{N}^{\prime \prime \prime}$ as follows:

$$
I_{N} U(x, y, z, t)=\sum_{j=1}^{N_{x}} \sum_{k=1}^{N_{y}} \sum_{m=1}^{N_{z}} U_{j, k, m} g_{j}(x) g_{k}(y) g_{m}(z),
$$

where $U_{j, k, m}=U\left(x_{j}, y_{k}, z_{m}, t\right)$ and its vector form is denoted by

$$
\boldsymbol{U}=\left(U_{1,1,1}, \cdots, U_{N_{x}, 1,1}, \cdots, U_{1, N_{y}, 1} \cdots, U_{N_{x}, N_{y}, 1} \cdots, U_{1, N_{y}, N_{z}}, \cdots, U_{N_{x}, N_{y}, N_{z}}\right)^{T} .
$$

Taking the first derivative with respect to $x, y$ and $z$, respectively, and evaluating the resulting expression at collocation points $\left(x_{j}, y_{k}, z_{m}\right)$, we can obtain

$$
\begin{aligned}
& \frac{\partial I_{N} U\left(x_{j}, y_{k}, z_{m}, t\right)}{\partial x}=\left[\left(\boldsymbol{I}_{N_{z}} \otimes \boldsymbol{I}_{N_{y}} \otimes \boldsymbol{D}_{1}^{x}\right) \boldsymbol{U}\right]_{N_{x} N_{y}(m-1)+N_{x}(k-1)+j} \\
& :=\left[\boldsymbol{D}_{1} \boldsymbol{U}\right]_{N_{x} N_{y}(m-1)+N_{x}(k-1)+j}, \\
& \frac{\partial I_{N} U\left(x_{j}, y_{k}, z_{m}, t\right)}{\partial y}=\left[\left(\boldsymbol{I}_{N_{z}} \otimes \boldsymbol{D}_{1}^{y} \otimes \boldsymbol{I}_{N_{x}}\right) \boldsymbol{U}\right]_{N_{x} N_{y}(m-1)+N_{x}(k-1)+j} \\
& :=\left[\boldsymbol{D}_{2} \boldsymbol{U}\right]_{N_{x} N_{y}(m-1)+N_{x}(k-1)+j}, \\
& \frac{\partial I_{N} U\left(x_{j}, y_{k}, z_{m}, t\right)}{\partial z}=\left[\left(\boldsymbol{D}_{1}^{z} \otimes \boldsymbol{I}_{N_{y}} \otimes \boldsymbol{I}_{N_{x}}\right) \boldsymbol{U}\right]_{N_{x} N_{y}(m-1)+N_{x}(k-1)+j} \\
& :=\left[\boldsymbol{D}_{3} \boldsymbol{U}\right]_{N_{x} N_{y}(m-1)+N_{x}(k-1)+j},
\end{aligned}
$$


where $\otimes$ is Kronecker product, $I_{N_{w}}$ is the identity matrix of dimension $N_{w} \times N_{w}$ and

$$
\left(\boldsymbol{D}_{1}^{w}\right)_{j_{1}, j_{2}}= \begin{cases}\frac{1}{2} \mu_{w}(-1)^{j_{1}+j_{2}} \cot \left(r_{w}\right), & j_{1} \neq j_{2}, \\ 0, & j_{1}=j_{2},\end{cases}
$$

with $r_{w}=\mu_{w} \frac{w_{j_{1}}-w_{j_{2}}}{2}, j_{1}, j_{2}=1, \cdots, N_{w}$. It is easy to show that the matrices $\boldsymbol{D}_{1}^{w}, \boldsymbol{D}_{1}, \boldsymbol{D}_{2}$, and $D_{3}$ have the following properties:

- $\boldsymbol{D}_{1}^{w}$ is skew-symmetric. Further, let

$$
\Lambda=\mathrm{i} \mu_{w} \operatorname{diag}\left(0,1, \cdots, \frac{N_{w}}{2}-1,0,-\frac{N_{w}}{2}+1, \cdots,-2,-1\right),
$$

we have

$$
\boldsymbol{D}_{1}^{w}=\mathcal{F}_{N_{w}}^{-1} \Lambda \mathcal{F}_{N_{w}}
$$

where $\mathcal{F}_{N_{w}}$ is the matrix of DFT coefficients with entries given by $\left(\mathcal{F}_{N_{w}}\right)_{j, k}=\omega_{N_{w}}^{-j, k}$, $\omega_{N_{w}}=e^{i \frac{2 \pi}{N_{w}}},\left(\mathcal{F}_{N_{w}}^{-1}\right)_{j, k}=\frac{1}{N_{w}} \omega_{N_{w}}^{j, k}$.

- Commutative law of multiplication

$$
\boldsymbol{D}_{s_{1}} \otimes \boldsymbol{D}_{s_{2}}=\boldsymbol{D}_{s_{2}} \otimes \boldsymbol{D}_{s_{1}}, s_{1}, s_{2}=1,2,3, \text { and } s_{1} \neq s_{2} .
$$

- Skew symmetry

$$
\boldsymbol{D}_{s_{3}}^{T}=-\boldsymbol{D}_{s_{3}}, \quad s_{3}=1,2,3 .
$$

We apply the Fourier pseudo-spectral method to (2.4) in space and obtain the semidiscrete system

$$
\frac{d}{d t}\left[\begin{array}{c}
H \\
E
\end{array}\right]=\left[\begin{array}{cc}
0 & -\boldsymbol{I} \\
\boldsymbol{I} & 0
\end{array}\right]\left[\begin{array}{c}
\nabla_{\boldsymbol{H}} \overline{\mathcal{H}} \\
\nabla_{\boldsymbol{E}} \overline{\mathcal{H}}
\end{array}\right]-\bar{\sigma}\left[\begin{array}{c}
\boldsymbol{H} \\
\boldsymbol{E}
\end{array}\right],
$$

where

$$
\overline{\mathcal{H}}=\frac{1}{2 \epsilon} \boldsymbol{H}^{T}(\mathbf{D H})+\frac{1}{2 \mu} \boldsymbol{E}^{T}(\boldsymbol{D E}),
$$

$\boldsymbol{I} \in \mathbb{R}^{3 d \times 3 d}, d=N_{x} \times N_{y} \times N_{z}$ and

$$
\boldsymbol{D}=\left[\begin{array}{ccc}
0 & -\boldsymbol{D}_{3} & \boldsymbol{D}_{2} \\
\boldsymbol{D}_{3} & 0 & -\boldsymbol{D}_{1} \\
-\boldsymbol{D}_{2} & \boldsymbol{D}_{1} & 0
\end{array}\right]
$$

is symmetric structure matrix corresponding to the discretization of the operator $\nabla \times$.

Remark 2.4. It should note that $\boldsymbol{H}=\left(\boldsymbol{H}_{x}^{T}, \boldsymbol{H}_{y}^{T}, \boldsymbol{H}_{z}^{T}\right)^{T}, \boldsymbol{E}=\left(\boldsymbol{E}_{x}^{T}, \boldsymbol{E}_{y}^{T}, \boldsymbol{E}_{z}^{T}\right)^{T}$ and the components of the vectors $\boldsymbol{H}_{w}$ and $\boldsymbol{E}_{w}$ are the values of grid functions, which are different from those in (2.1). Without being confused, the notations $\boldsymbol{H}$ and $\boldsymbol{E}$ are still be adopted in the subsequent sections. 


\subsection{Conformal Fourier pseudo-spectral scheme}

The system (2.16) is first split as

$$
\frac{d}{d t}\left[\begin{array}{c}
H \\
E
\end{array}\right]=\left[\begin{array}{cc}
0 & -I \\
I & 0
\end{array}\right]\left[\begin{array}{c}
\epsilon^{-1} D H \\
\mu^{-1} D E
\end{array}\right],
$$

and

$$
\frac{d}{d t}\left[\begin{array}{c}
H \\
E
\end{array}\right]=-\bar{\sigma}\left[\begin{array}{c}
H \\
E
\end{array}\right] .
$$

Then, we solve (2.19) by a second order energy-conserved method in time

$$
\begin{aligned}
& \frac{\boldsymbol{H}^{n+1}-\boldsymbol{H}^{n}}{\tau}=-\mu^{-1} \boldsymbol{D} \frac{\boldsymbol{E}^{n+1}+\boldsymbol{E}^{n}}{2}, \\
& \frac{\boldsymbol{E}^{n+1}-\boldsymbol{E}^{n}}{\tau}=\epsilon^{-1} \boldsymbol{D} \frac{\boldsymbol{H}^{n+1}+\boldsymbol{H}^{n}}{2} .
\end{aligned}
$$

Eqs. (2.20) are integrated exactly

$$
\boldsymbol{H}(t)=e^{-\bar{\sigma} t} \boldsymbol{H}(0), \quad \boldsymbol{E}(t)=e^{-\bar{\sigma} t} \boldsymbol{E}(0) .
$$

Finally, by virtue of the Strang splitting, which was firstly proposed in Ref. [31], and further developed and analyzed in Refs. $[17,26,28]$, we can obtain

$$
\begin{aligned}
\frac{e^{\frac{\bar{\sigma} \tau}{2}} \boldsymbol{H}^{n+1}-e^{-\frac{\bar{\sigma} \tau}{2}} \boldsymbol{H}^{n}}{\tau} & =-\mu^{-1} \boldsymbol{D} \frac{e^{\frac{\bar{\sigma} \tau}{2}} \boldsymbol{E}^{n+1}+e^{-\frac{\bar{\sigma} \tau}{2}} \boldsymbol{E}^{n}}{2}, \\
\frac{e^{\frac{\bar{\sigma} \tau}{2}} \boldsymbol{E}^{n+1}-e^{-\frac{\bar{\sigma} \tau}{2}} \boldsymbol{E}^{n}}{\tau} & =\varepsilon^{-1} \boldsymbol{D} \frac{e^{\frac{\bar{\sigma} \tau}{2}} \boldsymbol{H}^{n+1}+e^{-\frac{\bar{\sigma} \tau}{2}} \boldsymbol{H}^{n}}{2},
\end{aligned}
$$

which is referred to as our conformal Fourier pseudo-spectral (CFP) scheme.

Remark 2.5. The conformal Hamiltonian system (2.4) is incorrect for the 2D transverseelectric (TE) waves in the PML medium, but the proposed scheme (2.24)-(2.25) are valid.

\section{Numerical analysis}

In this section, we first show that the scheme (2.24)-(2.25) can preserve the discrete conformal symplectic conservation law and the five discrete conformal energy conservation laws. Then, an optimal error estimate of the scheme (2.24)-(2.25) is established in discrete $L^{2}$-norm. 


\subsection{Conservation of the scheme}

Let $\left\{U_{j, k, m}^{n}, V_{j, k, m}^{n} \mid j=1, \cdots, N_{x}, k=1, \cdots, N_{y}, m=1, \cdots, N_{z}, 0 \leqslant n \leqslant M\right\}$ be two mesh functions defined on $\Omega_{h \tau}$. We define the discrete inner products and norms by

$$
\left\langle\boldsymbol{U}^{n}, \boldsymbol{V}^{n}\right\rangle_{h}=\sum_{j=1}^{N_{x}} \sum_{k=1}^{N_{y}} \sum_{m=1}^{N_{z}} U_{j, k, m} V_{j, k, m} h_{x} h_{y} h_{z}, \quad\left\|\boldsymbol{U}^{n}\right\|_{h}^{2}=\left\langle\boldsymbol{U}^{n}, \boldsymbol{U}^{n}\right\rangle_{h}
$$

For vectors $\boldsymbol{U}^{n}=\left[\left(\boldsymbol{U}_{x}^{n}\right)^{T},\left(\boldsymbol{U}_{y}^{n}\right)^{T},\left(\boldsymbol{U}_{z}^{n}\right)^{T}\right]^{T}$ and $\boldsymbol{V}^{n}=\left[\left(\boldsymbol{V}_{x}^{n}\right)^{T},\left(\boldsymbol{V}_{y}^{n}\right)^{T},\left(\boldsymbol{V}_{z}^{n}\right)^{T}\right]^{T}$, the corresponding inner product and discrete norms are

$$
\begin{aligned}
& \left\langle\boldsymbol{U}^{n}, \boldsymbol{V}^{n}\right\rangle_{h}=\left\langle\boldsymbol{U}_{x}^{n}, \boldsymbol{V}_{x}^{n}\right\rangle_{h}+\left\langle\boldsymbol{U}_{y}^{n}, \boldsymbol{V}_{y}^{n}\right\rangle_{h}+\left\langle\boldsymbol{U}_{z}^{n}, \boldsymbol{V}_{z}^{n}\right\rangle_{h}, \\
& \left\|\boldsymbol{U}^{n}\right\|_{h}^{2}=\left\langle\boldsymbol{U}_{x}^{n}, \boldsymbol{U}_{x}^{n}\right\rangle_{h}+\left\langle\boldsymbol{U}_{y}^{n}, \boldsymbol{U}_{y}^{n}\right\rangle_{h}+\left\langle\boldsymbol{U}_{z}^{n}, \boldsymbol{U}_{z}^{n}\right\rangle_{h} .
\end{aligned}
$$

Further, we define the following semi-norms

$$
\begin{aligned}
& \left\|\boldsymbol{U}^{n}\right\|_{\boldsymbol{D}_{1}^{x}, h}=\left\langle\boldsymbol{D}_{1} \boldsymbol{U}^{n}, \boldsymbol{D}_{1} \boldsymbol{U}^{n}\right\rangle_{h}^{1 / 2}, \quad\left\|\boldsymbol{U}^{n}\right\|_{\boldsymbol{D}_{1}^{y}, h}=\left\langle\boldsymbol{D}_{2} \boldsymbol{U}^{n}, \boldsymbol{D}_{2} \boldsymbol{U}^{n}\right\rangle_{h}^{1 / 2}, \\
& \left\|\boldsymbol{U}^{n}\right\|_{\boldsymbol{D}_{1}^{z}, h}=\left\langle\boldsymbol{D}_{3} \boldsymbol{U}^{n}, \boldsymbol{D}_{3} \boldsymbol{U}^{n}\right\rangle_{h}^{1 / 2},
\end{aligned}
$$

for grid function and

$$
\left\|\boldsymbol{U}^{n}\right\|_{\boldsymbol{D}_{1}^{w}, h}^{2}=\left\|\boldsymbol{U}_{x}^{n}\right\|_{\boldsymbol{D}_{1}^{w}, h}^{2}+\left\|\boldsymbol{U}_{y}^{n}\right\|_{\boldsymbol{D}_{1}^{w}, h}^{2}+\left\|\boldsymbol{U}_{z}^{n}\right\|_{\boldsymbol{D}_{1}^{w}, h}^{2}
$$

for grid vector function $\boldsymbol{U}^{n}=\left[\left(\boldsymbol{U}_{x}^{n}\right)^{T},\left(\boldsymbol{U}_{y}^{n}\right)^{T},\left(\boldsymbol{U}_{z}^{n}\right)^{T}\right]^{T}$.

Theorem 3.1. The solutions $\boldsymbol{H}^{n}$ and $\boldsymbol{E}^{n}$ of (2.24)-(2.25) can preserve the discrete conformal symplectic structure

$$
\omega^{n+1}=e^{-2 \bar{\sigma} \tau} \omega^{n}, \quad \omega^{n}=d \boldsymbol{E}^{n} \wedge d \boldsymbol{H}^{n}, \quad n=0, \cdots, M-1,
$$

where

$$
\begin{aligned}
& d \boldsymbol{E}^{n} \wedge d \boldsymbol{H}^{n}=d \boldsymbol{E}_{x}^{n} \wedge d \boldsymbol{H}_{x}^{n}+d \boldsymbol{E}_{y}^{n} \wedge d \boldsymbol{H}_{y}^{n}+d \boldsymbol{E}_{z}^{n} \wedge d \boldsymbol{H}_{z}^{n}, \\
& d \boldsymbol{E}_{w}^{n} \wedge d \boldsymbol{H}_{w}^{n}=\sum_{j=1}^{N_{x}} \sum_{k=1}^{N_{y}} \sum_{m=1}^{N_{z}} d \boldsymbol{E}_{w_{j, k, m}}^{n} \wedge d \boldsymbol{H}_{w_{j, k, m}}^{n} .
\end{aligned}
$$

Proof. We rewrite (2.24)-(2.25) as

$$
\left[\begin{array}{cc}
0 & \boldsymbol{I} \\
-\boldsymbol{I} & 0
\end{array}\right]\left[\begin{array}{c}
\frac{e^{\frac{\bar{\sigma} \tau}{2}} H^{n+1}-e^{-\frac{\bar{\sigma} \tau}{2}} \boldsymbol{H}^{n}}{\tau} \\
\frac{e^{\frac{\bar{\sigma} \tau}{2}} E^{n+1}-e^{-\frac{\bar{\sigma} \tau}{2}} E^{n}}{\tau}
\end{array}\right]=\left[\begin{array}{cc}
\boldsymbol{\epsilon}^{-1} \boldsymbol{D} & 0 \\
0 & \mu^{-1} \boldsymbol{D}
\end{array}\right]\left[\begin{array}{c}
\frac{e^{\frac{\bar{\sigma} \tau}{2}} \boldsymbol{H}^{n+1}+e^{-\frac{\bar{\sigma} \tau}{2}} \boldsymbol{H}^{n}}{2} \\
\frac{e^{\frac{\bar{\sigma} \tau}{2}} E^{n+1}+e^{-\frac{\bar{\sigma} \tau}{2}} E^{n}}{2}
\end{array}\right]
$$


The variational equations associated with (3.2) read

$$
\left[\begin{array}{cc}
0 & \boldsymbol{I} \\
-\boldsymbol{I} & 0
\end{array}\right]\left[\begin{array}{c}
\frac{e^{\frac{\bar{\sigma} \tau}{2}} d H^{n+1}-e^{-\frac{\bar{\sigma} \tau}{2}} d \boldsymbol{H}^{n}}{\tau} \\
\frac{e^{\frac{\sigma \tau \tau}{2}} d \boldsymbol{E}^{n+1}-e^{-\frac{\bar{\sigma} \tau}{2}} d \boldsymbol{E}^{n}}{\tau}
\end{array}\right]=\left[\begin{array}{cc}
\epsilon^{-1} \boldsymbol{D} & 0 \\
0 & \mu^{-1} \boldsymbol{D}
\end{array}\right]\left[\begin{array}{c}
\frac{e^{\frac{\bar{\sigma} \tau}{2}} d H^{n+1}+e^{-\frac{\bar{\sigma} \tau}{2}} d H^{n}}{2} \\
\frac{e^{\frac{\bar{\sigma} \tau}{2}} d \boldsymbol{E}^{n+1}+e^{-\frac{\bar{\sigma} \tau}{2}} d \boldsymbol{E}^{n}}{2}
\end{array}\right]
$$

Taking the discrete wedge product of (3.3) with $\left[\frac{\frac{\frac{\frac{\sigma}{}}{2} d}{d H^{n+1}+e^{-\frac{\partial \tau}{2}} d H^{n}}}{2}\right]$ and noting the fact

$$
\left[\begin{array}{c}
\frac{e^{\frac{\bar{\sigma} \tau}{2}} d H^{n+1}+e^{-\frac{\bar{\sigma} \tau}{2}} d H^{n}}{2} \\
\frac{e^{\frac{\bar{\sigma} \tau}{2}} d \boldsymbol{E}^{n+1}+e^{-\frac{\bar{\sigma} \tau}{2}} d \boldsymbol{E}^{n}}{2}
\end{array}\right] \wedge\left[\begin{array}{cc}
\epsilon^{-1} \boldsymbol{D} & 0 \\
0 & \mu^{-1} \boldsymbol{D}
\end{array}\right]\left[\begin{array}{c}
\frac{e^{\frac{\bar{\sigma} \tau}{2}} d \boldsymbol{H}^{n+1}+e^{-\frac{\bar{\sigma} \tau}{2}} d H^{n}}{2} \\
\frac{e^{\frac{\bar{\sigma} \tau}{2}} d \boldsymbol{E}^{n+1}+e^{-\frac{\bar{\sigma} \tau}{2}} d \boldsymbol{E}^{n}}{2}
\end{array}\right]=0
$$

we finish the proof.

Theorem 3.2. The solutions $\boldsymbol{H}^{n}$ and $\boldsymbol{E}^{n}$ of (2.24)-(2.25) satisfy the discrete conformal energy conservation law

$$
\mathcal{E}_{1}^{n+1}=e^{-2 \bar{\sigma} \tau} \mathcal{E}_{1}^{n}, \quad \mathcal{E}_{1}^{n}=\frac{1}{2 \epsilon}\left\langle\boldsymbol{H}^{n}, \boldsymbol{D} \boldsymbol{H}^{n}\right\rangle_{h}+\frac{1}{2 \mu}\left\langle\boldsymbol{E}^{n}, \mathbf{D} \boldsymbol{E}^{n}\right\rangle_{h}, \quad n=0, \cdots, M-1,
$$

Proof. We rewrite (2.24)-(2.25) as

$$
\begin{aligned}
{\left[\begin{array}{c}
\frac{e^{\frac{\bar{\sigma} \tau}{2}} H^{n+1}-e^{-\frac{\bar{\sigma} \tau}{2}} H^{n}}{\tau} \\
\frac{e^{\frac{\sigma \tau \tau}{2}} E^{n+1}-e^{-\frac{\bar{\sigma} \tau}{2}} E^{n}}{\tau}
\end{array}\right] } & =\left[\begin{array}{cc}
0 & -\boldsymbol{D} \\
\boldsymbol{D} & 0
\end{array}\right]\left[\begin{array}{c}
\frac{e^{\frac{\bar{\sigma} \tau}{2}} H^{n+1}+e^{-\frac{\bar{\sigma} \tau}{2}} H^{n}}{2 \epsilon} \\
\frac{e^{\frac{\bar{\sigma} \tau}{2}} E^{n+1}+e^{-\frac{\tilde{\sigma} \tau}{2}} E^{n}}{2 \mu}
\end{array}\right] \\
& =\left[\begin{array}{cc}
0 & -\boldsymbol{I} \\
\boldsymbol{I} & 0
\end{array}\right]\left[\begin{array}{c}
\boldsymbol{D} \frac{e^{\frac{\tilde{\sigma} \tau}{2}} H^{n+1}+e^{-\frac{\bar{\sigma} \tau}{2}} H^{n}}{2 \epsilon} \\
\boldsymbol{D} \frac{e^{\frac{\bar{\sigma} \tau}{2}} E^{n+1}+e^{-\frac{\bar{\sigma} \tau}{2}} E^{n}}{2 \mu}
\end{array}\right] .
\end{aligned}
$$

Taking the inner product of (3.6) with $\left[\begin{array}{c}D \frac{e^{\frac{\bar{\sigma} \tau}{2}} H^{n+1}+e^{-\frac{\bar{\sigma} \tau}{2}} H^{n}}{2 \epsilon} \\ D \frac{e^{\frac{\bar{\sigma} \tau}{2}} E^{n+1}+e^{-\frac{\bar{\sigma} \tau}{2}} E^{n}}{2 \mu}\end{array}\right]$, we can obtain (3.5).

Theorem 3.3. The solutions $\boldsymbol{H}^{n}$ and $\boldsymbol{E}^{n}$ of (2.24)-(2.25) satisfy the discrete conformal energy conservation law

$$
\mathcal{E}_{2}^{n+1}=e^{-2 \bar{\sigma} \tau} \mathcal{E}_{2}^{n}, \quad \mathcal{E}_{2}^{n}=\frac{\mu}{2}\left\|\boldsymbol{H}^{n}\right\|_{h}^{2}+\frac{\epsilon}{2}\left\|E^{n}\right\|_{h}^{2}, \quad n=0, \cdots, M-1 .
$$

Proof. Making the inner product of (3.6) with $\left[\begin{array}{c}\mu \frac{\frac{e^{\frac{\sigma}{2}}}{2} H^{n+1}+e^{-\frac{\bar{\sigma} \tau}{2}} H^{n}}{2} \\ \epsilon \frac{e^{\frac{\bar{\sigma} \tau}{2}} E^{n+1}+e^{-\frac{\bar{\sigma} \tau}{2}} E^{n}}{2}\end{array}\right]$, we can finish the proof.

From Theorem 3.3, we can obtain the following stability theorem. 
Corollary 3.1. The proposed scheme (2.24)-(2.25) is unconditionally stable.

Theorem 3.4. The solutions $\boldsymbol{H}^{n}$ and $\boldsymbol{E}^{n}$ of (2.24)-(2.25) satisfy the discrete conformal energy conservation law

$$
\mathcal{E}_{3}^{n+1}=e^{-2 \bar{\sigma} \tau} \mathcal{E}_{3}^{n}, \quad \mathcal{E}_{3}^{n}=\frac{\mu}{2}\left\|\hat{\delta}_{t} H^{n-1 / 2}\right\|_{h}^{2}+\frac{\epsilon}{2}\left\|\hat{\delta}_{t} \boldsymbol{E}^{n-1 / 2}\right\|_{h}^{2}, \quad n=1, \cdots, M-1,
$$

where $\hat{\delta}_{t} \boldsymbol{E}^{n-1 / 2}=\frac{\boldsymbol{E}^{n}-\boldsymbol{E}^{n-1}}{\tau}$ and $\hat{\delta}_{t} \boldsymbol{H}^{n-1 / 2}=\frac{\boldsymbol{H}^{n}-\boldsymbol{H}^{n-1}}{\tau}$.

Proof. By simple calculations, we can deduce from (2.24)-(2.25) that

$$
\left[\begin{array}{c}
\mu \frac{e^{\frac{\sigma \tau}{2}} \hat{\delta}_{t} \boldsymbol{H}^{n+\frac{1}{2}}-e^{-\frac{\bar{\sigma} \tau}{2}} \hat{\delta}_{t} \boldsymbol{H}^{n-\frac{1}{2}}}{\tau} \\
\epsilon \frac{e^{\frac{\bar{\sigma} \tau}{2}} \hat{\delta}_{t} \boldsymbol{E}^{n+\frac{1}{2}}-e^{-\frac{\bar{\tau} \tau}{2}} \hat{\delta}_{t} \boldsymbol{E}^{n-\frac{1}{2}}}{\tau}
\end{array}\right]=\left[\begin{array}{cc}
0 & -\boldsymbol{D} \\
\boldsymbol{D} & 0
\end{array}\right]\left[\begin{array}{c}
\frac{e^{\frac{\bar{\sigma} \tau}{2}} \hat{\delta}_{t} \boldsymbol{H}^{n+\frac{1}{2}}+e^{-\frac{\bar{\sigma} \tau}{2}} \hat{\delta}_{t} \boldsymbol{H}^{n-\frac{1}{2}}}{2} \\
\frac{e^{\frac{\bar{\sigma} \tau}{2}} \hat{\delta}_{t} \boldsymbol{E}^{n+\frac{1}{2}}+e^{-\frac{\bar{\sigma} \tau}{2}} \hat{\delta}_{t} \boldsymbol{E}^{n-\frac{1}{2}}}{2}
\end{array}\right]
$$

Computing the inner product of (3.9) with $\left[\begin{array}{c}\frac{e^{\frac{\sigma}{2}} \hat{\delta}_{t} H^{n+\frac{1}{2}}+e^{-\frac{\tilde{\sigma} \tau}{2}} \hat{\delta}_{t} H^{n-\frac{1}{2}}}{2} \\ \frac{e^{\frac{\tilde{\sigma}}{2}} \hat{\delta}_{t} E^{n+\frac{1}{2}}+e^{-\frac{\bar{\sigma} \tau}{2}} \hat{\delta}_{t} E^{n-\frac{1}{2}}}{2}\end{array}\right]$, we finish the proof.

Let block diagonal matrices

$$
M_{x}=\left[\begin{array}{lll}
\boldsymbol{D}_{1} & & \\
& \boldsymbol{D}_{1} & \\
& & \boldsymbol{D}_{1}
\end{array}\right], \quad M_{y}=\left[\begin{array}{lll}
\boldsymbol{D}_{2} & & \\
& \boldsymbol{D}_{2} & \\
& & \boldsymbol{D}_{2}
\end{array}\right], \quad M_{z}=\left[\begin{array}{lll}
\boldsymbol{D}_{3} & & \\
& \boldsymbol{D}_{3} & \\
& & \boldsymbol{D}_{3}
\end{array}\right] .
$$

It follows from (2.14) that $M_{w} \boldsymbol{D}=\boldsymbol{D} M_{w}$. Thus, we can see from (2.24)-(2.25) that

$$
\left[\begin{array}{c}
\mu \frac{e^{\frac{\tilde{\sigma} \tau}{2}} M_{w} H^{n+1}-e^{-\frac{\bar{\sigma} \tau}{2}} M_{w} H^{n}}{\tau} \\
\epsilon \frac{e^{\frac{\sigma \tau}{2}} M_{w} E^{n+1}-e^{-\frac{\bar{\sigma} \tau}{2}} M_{w} E^{n}}{\tau}
\end{array}\right]=\left[\begin{array}{cc}
0 & -\boldsymbol{D} \\
\boldsymbol{D} & 0
\end{array}\right]\left[\begin{array}{c}
\frac{e^{\frac{\tilde{\sigma} \tau}{2}} M_{w} H^{n+1}+e^{-\frac{\bar{\sigma} \tau}{2}} M_{w} H^{n}}{2} \\
\frac{e^{\frac{\tilde{\sigma} \tau}{2}} M_{w} E^{n+1}+e^{-\frac{\bar{\sigma} \tau}{2}} M_{w} E^{n}}{2}
\end{array}\right]
$$

By the similar argument to Theorems 3.3 and 3.4, we can deduce the following conformal energy-conserved laws from (3.10).

Theorem 3.5. The solutions $\boldsymbol{H}^{n}$ and $\boldsymbol{E}^{n}$ of (2.24)-(2.25) possess the discrete conformal energy conservation laws

$$
\mathcal{E}_{4_{w}}^{n+1}=e^{-2 \bar{\sigma} \tau} \mathcal{E}_{4_{w}}^{n}, \quad \mathcal{E}_{4_{w}}^{n}=\mu\left\|\boldsymbol{H}^{n}\right\|_{D_{1}^{w w}, h}^{2}+\epsilon\left\|\boldsymbol{E}^{n}\right\|_{\boldsymbol{D}_{1}^{w}, h^{\prime}}^{2} \quad n=0, \cdots, M-1,
$$

and

$$
\mathcal{E}_{5_{w}}^{n+1}=e^{-2 \bar{\sigma} \tau} \mathcal{E}_{5_{w}}^{n}, \quad \mathcal{E}_{5_{w}}^{n}=\mu\left\|\hat{\delta}_{t} \boldsymbol{H}^{n-1 / 2}\right\|_{D_{1}^{w}, h}^{2}+\epsilon\left\|\hat{\delta}_{t} \boldsymbol{E}^{n-1 / 2}\right\|_{D_{1}^{w}, h^{\prime}}^{2} \quad n=1, \cdots, M-1 .
$$




\subsection{Error estimate}

In this subsection, we will establish an optimal error estimate for the CFP scheme. For simplicity, we only consider the cuboid domain $\Omega=[0,2 \pi]^{3}$, more general cuboid domain can be translated into $\Omega$. For any positive integer $r$, the semi-norm and the norm of $H^{r}(\Omega)$ are denoted by $|\cdot|_{r}$ and $\|\cdot\|_{r}$ respectively. $\|\cdot\|_{0}$ is denoted by $\|\cdot\|$ for sake of brevity. Let $C_{p}^{\infty}$ be the set of infinitely differentiable functions with period $2 \pi$ defined on $\Omega$ for all variables, and $H_{p}^{r}(\Omega)$ is the closure of $C_{p}^{\infty}$ in $H^{r}(\Omega)$. Let $N_{x}=N_{y}=N_{z}=N$ (i.e., $h_{x}=h_{y}=$ $\left.h_{z}=h\right)$, the interpolation space $S_{N}^{\prime \prime \prime}$ can be written as

$$
\begin{gathered}
S_{N}^{\prime \prime \prime}=\left\{u \mid u=\sum_{|j|,|k|,|m| \leq \frac{N}{2}} \frac{\hat{u}_{j, k, m}}{c_{j} c_{k} c_{m}} e^{\mathrm{i}(j x+k y+m z)}: \hat{u}_{\frac{N}{2}, k, m}=\hat{u}_{-\frac{N}{2}, k, m}\right. \\
\left.\hat{u}_{j, \frac{N}{2}, m}=\hat{u}_{j,-\frac{N}{2}, m}, \hat{u}_{j, k, \frac{N}{2}}=\hat{u}_{j, k,-\frac{N}{2}}\right\},
\end{gathered}
$$

where $c_{l}=1,|l|<\frac{N}{2}, c_{-\frac{N}{2}}=c_{\frac{N}{2}}=2$.

We introduce the projection space

$$
S_{N}=\left\{u \mid u=\sum_{|j|,|k|,|m| \leq \frac{N}{2}} \hat{u}_{j, k, m} e^{\mathrm{i}(j x+k y+m z)}\right\} .
$$

It is obvious that $S_{N-2} \subseteq S_{N}^{\prime \prime \prime} \subseteq S_{N}$. We denote $P_{N}: L^{2}(\Omega) \rightarrow S_{N}$ as the orthogonal projection operator and recall the interpolation operator $I_{N}: C(\Omega) \rightarrow S_{N}^{\prime \prime \prime}$. It is easy to see that $P_{N}$ and $I_{N}$ satisfy [16]:

$$
\begin{aligned}
& \text { 1. } P_{N} \partial_{w} u=\partial_{w} P_{N} u, \quad I_{N} \partial_{w} u \neq \partial_{w} I_{N} u ; \\
& \text { 2. } P_{N} u=u, \forall u \in S_{N}, \quad I_{N} u=u, \forall u \in S_{N}^{\prime \prime \prime} .
\end{aligned}
$$

Lemma 3.1. [7] For $\boldsymbol{u} \in\left[S_{N}^{\prime \prime \prime}\right]^{3},\|\boldsymbol{u}\| \leqslant\|\boldsymbol{u}\|_{h} \leqslant 2 \sqrt{2}\|\boldsymbol{u}\|$.

Lemma 3.2. [9] If $0 \leqslant \alpha \leqslant r$ and $\boldsymbol{u} \in\left[H_{p}^{r}(\Omega)\right]^{3}$, then

$$
\left\|P_{N} \boldsymbol{u}-\boldsymbol{u}\right\|_{\alpha} \leqslant C N^{\alpha-r}|\boldsymbol{u}|_{r}
$$

and in addition if $r>3 / 2$ then

$$
\left\|I_{N} \boldsymbol{u}-\boldsymbol{u}\right\|_{\alpha} \leqslant C N^{\alpha-r}|\boldsymbol{u}|_{r}
$$

Theorem 3.6. We assume that the exact solutions $(\boldsymbol{H}, \boldsymbol{E})$ of (2.3) are smooth enough: $e^{\bar{\sigma} t} \boldsymbol{H}, e^{\bar{\sigma} t} \boldsymbol{E} \in$ $C^{3}\left(0, T ;\left[H_{p}^{r}(\Omega)\right]^{3}\right), r>\frac{3}{2}$. Then, there exists a positive constant $C$ independent of $\tau$ and $h$, such that the numerical solutions $\left(\boldsymbol{H}^{n}, \boldsymbol{E}^{n}\right)$ of (2.24)-(2.25) satisfy

$$
\left(\mu\left\|\boldsymbol{H}\left(t_{n}\right)-\boldsymbol{H}^{n}\right\|_{h}^{2}+\epsilon\left\|\boldsymbol{E}\left(t_{n}\right)-\boldsymbol{E}^{n}\right\|_{h}^{2}\right)^{\frac{1}{2}} \leqslant C T e^{-\bar{\sigma} t_{n}}\left(\tau^{2}+N^{-r}\right), \quad 0 \leqslant n \leqslant M .
$$


Proof. We rewrite (2.3) as

$$
\mu \partial_{t}\left(e^{\bar{\sigma} t} \boldsymbol{H}\right)+\nabla \times\left(e^{\bar{\sigma} t} \boldsymbol{E}\right)=0, \quad \epsilon \partial_{t}\left(e^{\bar{\sigma} t} \boldsymbol{E}\right)-\nabla \times\left(e^{\bar{\sigma} t} \boldsymbol{H}\right)=0 .
$$

Let

$$
\boldsymbol{E}^{*}=P_{N-2} \boldsymbol{E}, \quad \boldsymbol{H}^{*}=P_{N-2} \boldsymbol{H},
$$

where $P_{N-2}$ denotes the orthogonal projection operator from $L^{2}(\Omega)$ to $S_{N-2}$. The projection equations of (3.18) are written as

$$
\mu \partial_{t}\left(e^{\bar{\sigma} t} \boldsymbol{H}^{*}\right)+\nabla \times\left(e^{\bar{\sigma} t} \boldsymbol{E}^{*}\right)=0, \quad \epsilon \partial_{t}\left(e^{\bar{\sigma} t} \boldsymbol{E}^{*}\right)-\nabla \times\left(e^{\bar{\sigma} t} \boldsymbol{H}^{*}\right)=0 .
$$

Let

$$
\begin{aligned}
& \boldsymbol{\xi}^{n+\frac{1}{2}}=\mu \frac{e^{\frac{\bar{\sigma} \tau}{2}}\left(\boldsymbol{H}^{*}\right)^{n+1}-e^{-\frac{\bar{\sigma} \tau}{2}}\left(\boldsymbol{H}^{*}\right)^{n}}{\tau}+\nabla \times \frac{e^{\frac{\bar{\sigma} \tau}{2}}\left(\boldsymbol{E}^{*}\right)^{n+1}+e^{-\frac{\bar{\sigma} \tau}{2}}\left(\boldsymbol{E}^{*}\right)^{n}}{2} \\
& \eta^{n+\frac{1}{2}}=\epsilon \frac{e^{\frac{\bar{\sigma} \tau}{2}}\left(\boldsymbol{E}^{*}\right)^{n+1}-e^{-\frac{\bar{\sigma} \tau}{2}}\left(\boldsymbol{E}^{*}\right)^{n}}{\tau}-\nabla \times \frac{e^{\frac{\bar{\sigma} \tau}{2}}\left(\boldsymbol{H}^{*}\right)^{n+1}+e^{-\frac{\bar{\sigma} \tau}{2}}\left(\boldsymbol{H}^{*}\right)^{n}}{2} .
\end{aligned}
$$

Making use of Lemma 3.2, (3.18), and the Taylor's expansion, we have

$$
\left\|\boldsymbol{\zeta}^{n+\frac{1}{2}}\right\| \leqslant C e^{-\bar{\sigma} t} n+\frac{1}{2}\left(\tau^{2}+N^{-r}\right), \quad n=0, \cdots, M-1
$$

By noting $\xi^{n+\frac{1}{2}} \in\left[S_{N}^{\prime \prime \prime}\right]^{3}$ and Lemma 3.1, we can prove that

$$
\left\|\boldsymbol{\xi}^{n+\frac{1}{2}}\right\|_{h} \leqslant C e^{-\bar{\sigma} t} n+\frac{1}{2}\left(\tau^{2}+N^{-r}\right)
$$

Similarly, we have

$$
\left\|\eta^{n+\frac{1}{2}}\right\|_{h} \leqslant C e^{-\bar{\sigma} t} t_{n+\frac{1}{2}}\left(\tau^{2}+N^{-r}\right), \quad n=0, \cdots, M-1 .
$$

Thus, it follows from (3.23) and (3.24) that

$$
\left(\left\|e^{\bar{\sigma} t} t_{n+\frac{1}{2}} \xi^{n+\frac{1}{2}}\right\|_{h}^{2}+\left\|e^{\bar{\sigma} t} n+\frac{1}{2} \eta^{n+\frac{1}{2}}\right\|_{h}^{2}\right)^{\frac{1}{2}} \leqslant C\left(\tau^{2}+N^{-r}\right),
$$

where the inequation $\sqrt{a^{2}+b^{2}} \leqslant a+b, \forall a, b \geqslant 0$ is used.

Note that if $\widetilde{U}^{*} \in S_{N}^{\prime \prime \prime}$, we have

$$
\begin{aligned}
& \partial_{x} \widetilde{U}^{*}\left(x_{j}, y_{k}, z_{m}\right)=\partial_{x}\left[I_{N} \widetilde{U}^{*}\left(x_{j}, y_{k}, z_{m}\right)\right]=\left[\boldsymbol{D}_{1} \widetilde{\boldsymbol{U}}^{*}\right]_{N_{x} N_{y}(m-1)+N_{x}(k-1)+j,} \\
& \partial_{y} \widetilde{U}^{*}\left(x_{j}, y_{k}, z_{m}\right)=\partial_{y}\left[I_{N} \widetilde{U}^{*}\left(x_{j}, y_{k}, z_{m}\right)\right]=\left[\boldsymbol{D}_{2} \widetilde{\boldsymbol{U}}^{*}\right]_{N_{x} N_{y}(m-1)+N_{x}(k-1)+j,} \\
& \partial_{z} \widetilde{U}^{*}\left(x_{j}, y_{k}, z_{m}\right)=\partial_{z}\left[I_{N} \widetilde{U}^{*}\left(x_{j}, y_{k}, z_{m}\right)\right]=\left[\boldsymbol{D}_{3} \widetilde{\boldsymbol{U}}^{*}\right]_{N_{x} N_{y}(m-1)+N_{x}(k-1)+j,}
\end{aligned}
$$


where

$$
\widetilde{\boldsymbol{U}}^{*}=\left(\widetilde{U}_{1,1,1}^{*}, \cdots, \widetilde{U}_{N_{x}, 1,1}^{*} \cdots, \widetilde{U}_{1, N_{y}, 1}^{*}, \cdots, \widetilde{U}_{N_{x}, N_{y}, 1}^{*}, \cdots, \widetilde{U}_{1, N_{y}, N_{z}}^{*} \cdots, \widetilde{U}_{N_{x}, N_{y}, N_{z}}^{*}\right)^{T} .
$$

Recall that $\boldsymbol{E}^{*}, \boldsymbol{H}^{*} \in S_{N^{\prime \prime \prime}}$, we can get

$$
\begin{aligned}
& \boldsymbol{\xi}^{n+\frac{1}{2}}=\mu \frac{e^{\frac{\bar{\sigma} \tau}{2}}\left(\boldsymbol{H}^{*}\right)^{n+1}-e^{-\frac{\bar{\sigma} \tau}{2}}\left(\boldsymbol{H}^{*}\right)^{n}}{\tau}+\boldsymbol{D} \frac{e^{\frac{\bar{\sigma} \tau}{2}}\left(\boldsymbol{E}^{*}\right)^{n+1}+e^{-\frac{\bar{\sigma} \tau}{2}}\left(\boldsymbol{E}^{*}\right)^{n}}{2}, \\
& \eta^{n+\frac{1}{2}}=\epsilon \frac{e^{\frac{\bar{\sigma} \tau}{2}}\left(\boldsymbol{E}^{*}\right)^{n+1}-e^{-\frac{\bar{\sigma} \tau}{2}}\left(\boldsymbol{E}^{*}\right)^{n}}{\tau}-\boldsymbol{D} \frac{e^{\frac{\bar{\sigma} \tau}{2}}\left(\boldsymbol{H}^{*}\right)^{n+1}+e^{-\frac{\bar{\sigma} \tau}{2}}\left(\boldsymbol{H}^{*}\right)^{n}}{2},
\end{aligned}
$$

where the components of the above vectors are the values of grid functions.

We define the error functions

$$
\boldsymbol{E}_{1}{ }^{n}=\left(\boldsymbol{H}^{*}\right)^{n}-\boldsymbol{H}^{n}, \boldsymbol{E}_{2}{ }^{n}=\left(\boldsymbol{E}^{*}\right)^{n}-\boldsymbol{E}^{n}, \quad n=0, \cdots, M .
$$

Subtracting (2.24)-(2.25) from (3.26)-(3.27) respectively, and we can obtain the error equations as follows

$$
\begin{aligned}
& \xi^{n+\frac{1}{2}}=\mu \frac{e^{\frac{\bar{\sigma} \tau}{2}} E_{1}^{n+1}-e^{-\frac{\bar{\sigma} \tau}{2}} E_{1}^{n}}{\tau}+D \frac{e^{\frac{\bar{\sigma} \tau}{2}} E_{2}^{n+1}+e^{-\frac{\bar{\sigma} \tau}{2}} E_{2}^{n}}{2}, \\
& \eta^{n+\frac{1}{2}}=\epsilon \frac{e^{\frac{\bar{\sigma} \tau}{2}} E_{2}{ }^{n+1}-e^{-\frac{\bar{\sigma} \tau}{2}} E_{2}{ }^{n}}{\tau}-D \frac{e^{\frac{\bar{\sigma} \tau}{2}} E_{1}{ }^{n+1}+e^{-\frac{\bar{\sigma} \tau}{2}} E_{1}{ }^{n}}{2} .
\end{aligned}
$$

Computing the inner product of (3.29) and (3.30) with $e^{\frac{\bar{\sigma} \tau}{2}} \boldsymbol{E}_{1}{ }^{n+1}+e^{-\frac{\bar{\sigma} \tau}{2}} \boldsymbol{E}_{1}{ }^{n}$ and $e^{\frac{\bar{\sigma} \tau}{2}} \boldsymbol{E}_{2}{ }^{n+1}+$ $e^{-\frac{\sigma \tau}{2}} E_{2}{ }^{n}$, respectively, and then adding them up, we can gain the following energy identity

$$
\begin{aligned}
& \left\|\sqrt{\mu} e^{\bar{\sigma} t_{n+1}} \boldsymbol{E}_{1}^{n+1}-\frac{\tau}{2 \sqrt{\mu}} e^{\bar{\sigma} t_{n+\frac{1}{2}}} \boldsymbol{\xi}^{n+\frac{1}{2}}\right\|_{h}^{2}+\left\|\sqrt{\epsilon} e^{\bar{\sigma} t_{n+1}} \boldsymbol{E}_{2}{ }^{n+1}-\frac{\tau}{2 \sqrt{\epsilon}} e^{\bar{\sigma} t_{n+\frac{1}{2}}} \boldsymbol{\eta}^{n+\frac{1}{2}}\right\|_{h}^{2} \\
= & \left\|\sqrt{\mu} e^{\bar{\sigma} t_{n}} \boldsymbol{E}_{1}^{n}+\frac{\tau}{2 \sqrt{\mu}} e^{\bar{\sigma} t_{n+\frac{1}{2}}} \boldsymbol{\xi}^{n+\frac{1}{2}}\right\|_{h}^{2}+\left\|\sqrt{\epsilon} e^{\bar{\sigma} t_{n}} \boldsymbol{E}_{2}{ }^{n}+\frac{\tau}{2 \sqrt{\epsilon}} e^{\bar{\sigma} t_{n+\frac{1}{2}}} \boldsymbol{\eta}^{n+\frac{1}{2}}\right\|_{h}^{2} .
\end{aligned}
$$

With the triangle inequality of the norm and (3.31), we can get

$$
\begin{aligned}
& \left(\left\|\sqrt{\mu} e^{\bar{\sigma} t_{n+1}} \boldsymbol{E}_{1}{ }^{n+1}\right\|_{h}^{2}+\left\|\sqrt{\epsilon} e^{\bar{\sigma} t_{n+1}} \boldsymbol{E}_{2}{ }^{n+1}\right\|_{h}^{2}\right)^{\frac{1}{2}} \leqslant\left(\left\|\sqrt{\mu} e^{\bar{\sigma} t_{n+1}} \boldsymbol{E}_{1}{ }^{n+1}-\frac{\tau}{2 \sqrt{\mu}} e^{\bar{\sigma} t_{n+\frac{1}{2}}} \boldsymbol{\xi}^{n+\frac{1}{2}}\right\|_{h}^{2}\right. \\
& \left.+\left\|\sqrt{\epsilon} e^{\bar{\sigma} t_{n+1}} \boldsymbol{E}_{2}{ }^{n+1}-\frac{\tau}{2 \sqrt{\epsilon}} e^{\bar{\sigma} t_{n+\frac{1}{2}}} \boldsymbol{\eta}^{n+\frac{1}{2}}\right\|_{h}^{2}\right)^{\frac{1}{2}}+\frac{\tau}{2}\left(\left\|\frac{1}{\sqrt{\mu}} e^{\bar{\sigma} t_{n+\frac{1}{2}}} \boldsymbol{\xi}^{n+\frac{1}{2}}\right\|_{h}^{2}+\left\|\frac{1}{\sqrt{\epsilon}} e^{\bar{\sigma} t_{n+\frac{1}{2}}} \boldsymbol{\eta}^{n+\frac{1}{2}}\right\|_{h}^{2}\right)^{\frac{1}{2}} \\
= & \left(\left\|\sqrt{\mu} e^{\bar{\sigma} t_{n}} \boldsymbol{E}_{1}{ }^{n}+\frac{\tau}{2 \sqrt{\mu}} e^{\bar{\sigma} t_{n+\frac{1}{2}}} \boldsymbol{\xi}^{n+\frac{1}{2}}\right\|_{h}^{2}+\left\|\sqrt{\epsilon} e^{\bar{\sigma} t_{n}} \boldsymbol{E}_{2}{ }^{n}+\frac{\tau}{2 \sqrt{\epsilon}} e^{\bar{\sigma} t_{n+\frac{1}{2}}} \boldsymbol{\eta}^{n+\frac{1}{2}}\right\|_{h}^{2}\right)^{\frac{1}{2}} \\
& +\frac{\tau}{2}\left(\left\|\frac{1}{\sqrt{\mu}} e^{\bar{\sigma} t_{n+\frac{1}{2}}} \boldsymbol{\xi}^{n+\frac{1}{2}}\right\|_{h}^{2}+\left\|\frac{1}{\sqrt{\epsilon}} e^{\bar{\sigma} t_{n+\frac{1}{2}}} \boldsymbol{\eta}^{n+\frac{1}{2}}\right\|_{h}^{2}\right)^{\frac{1}{2}} \\
\leqslant & \left(\left\|\sqrt{\mu} e^{\bar{\sigma} t_{n}} \boldsymbol{E}_{1}{ }^{n}\right\|_{h}^{2}+\left\|\sqrt{\epsilon} e^{\bar{\sigma} t_{n}} \boldsymbol{E}_{2}{ }^{n}\right\|_{h}^{2}\right)^{\frac{1}{2}}+\tau\left(\left\|\frac{1}{\sqrt{\mu}} e^{\bar{\sigma} t_{n+\frac{1}{2}}} \boldsymbol{\xi}^{n+\frac{1}{2}}\right\|_{h}^{2}+\left\|\frac{1}{\sqrt{\epsilon}} e^{\bar{\sigma} t_{n+\frac{1}{2}}} \boldsymbol{\eta}^{n+\frac{1}{2}}\right\|_{h}^{2}\right)^{\frac{1}{2}} .
\end{aligned}
$$


Recursively, applying the above inequality from time level $n$ to 0 , we obtain

$$
\begin{aligned}
& \left(\left\|\sqrt{\mu} e^{\bar{\sigma} t_{n}} \boldsymbol{E}_{1}{ }^{n}\right\|_{h}^{2}+\left\|\sqrt{\epsilon} e^{\bar{\sigma} t_{n}} \boldsymbol{E}_{2}{ }^{n}\right\|_{h}^{2}\right)^{\frac{1}{2}} \\
\leqslant & \left(\left\|\sqrt{\mu} \boldsymbol{E}_{1}{ }^{0}\right\|_{h}^{2}+\left\|\sqrt{\epsilon} \boldsymbol{E}_{2}{ }^{0}\right\|_{h}^{2}\right)^{\frac{1}{2}}+\sum_{l=0}^{n-1} \tau\left(\left\|\frac{1}{\sqrt{\mu}} e^{\bar{\sigma} t_{l+\frac{1}{2}}} \boldsymbol{\xi}^{l+\frac{1}{2}}\right\|_{h}^{2}+\left\|\frac{1}{\sqrt{\epsilon}} e^{\bar{\sigma} t_{l+\frac{1}{2}}} \boldsymbol{\eta}^{l+\frac{1}{2}}\right\|_{h}^{2}\right)^{\frac{1}{2}} .
\end{aligned}
$$

With the use of $\boldsymbol{H}^{0}=\boldsymbol{H}(0), \boldsymbol{E}^{0}=\boldsymbol{E}(0)$, we can prove

$$
\begin{aligned}
\left\|\boldsymbol{E}_{1}^{0}\right\|_{h} & =\left\|\boldsymbol{H}(0)-\boldsymbol{H}^{*}(0)\right\|_{h} \\
& =\left\|I_{N}\left(\boldsymbol{H}(0)-\boldsymbol{H}(0)^{*}\right)\right\|_{h} \\
& \leqslant 2 \sqrt{2}\left\|I_{N}\left(\boldsymbol{H}(0)-\boldsymbol{H}(0)^{*}\right)\right\| \\
& \leqslant 2 \sqrt{2}\left(\left\|I_{N} \boldsymbol{H}(0)-\boldsymbol{H}(0)\right\|+\left\|\boldsymbol{H}(0)-\boldsymbol{H}(0)^{*}\right\|\right) \leqslant C N^{-r},
\end{aligned}
$$

and

$$
\begin{aligned}
\left\|\boldsymbol{E}_{2}{ }^{0}\right\|_{h} & =\left\|\boldsymbol{E}(0)-\boldsymbol{E}^{*}(0)\right\|_{h} \\
& =\left\|I_{N}\left(\boldsymbol{E}(0)-\boldsymbol{E}(0)^{*}\right)\right\|_{h} \\
& \leqslant 2 \sqrt{2}\left\|I_{N}\left(\boldsymbol{E}(0)-\boldsymbol{E}(0)^{*}\right)\right\| \\
& \leqslant 2 \sqrt{2}\left(\left\|I_{N} \boldsymbol{E}(0)-\boldsymbol{E}(0)\right\|+\left\|\boldsymbol{E}(0)-\boldsymbol{E}(0)^{*}\right\|\right) \leqslant C N^{-r},
\end{aligned}
$$

which further imply that

$$
\left(\left\|\sqrt{\mu} \boldsymbol{E}_{1}^{0}\right\|_{h}^{2}+\left\|\sqrt{\epsilon} \boldsymbol{E}_{2}^{0}\right\|_{h}^{2}\right)^{\frac{1}{2}} \leqslant C N^{-r},
$$

where Lemmas 3.1 and 3.2 are used.

By noting $n \tau<T$ and we can deduce from (3.25), (3.32) and (3.33) that

$$
\left(\left\|\sqrt{\mu} E_{1}{ }^{n}\right\|_{h}^{2}+\left\|\sqrt{\epsilon} E_{2}{ }^{n}\right\|_{h}^{2}\right)^{\frac{1}{2}} \leqslant C T e^{-\bar{\sigma} t_{n}}\left(\tau^{2}+N^{-r}\right) .
$$

Making use of (3.34) and the inequation, $a+b \leqslant \sqrt{2 a^{2}+2 b^{2}}, \forall a, b \geqslant 0$, we can obtain

$$
\left\|\sqrt{\mu} \boldsymbol{E}_{1}{ }^{n}\right\|_{h}+\left\|\sqrt{\epsilon} \boldsymbol{E}_{2}{ }^{n}\right\|_{h} \leqslant C T e^{-\bar{\sigma} t_{n}}\left(\tau^{2}+N^{-r}\right) .
$$

With Lemmas 3.1, 3.2 and (3.35), the following error estimate can be established

$$
\begin{aligned}
& \left(\mu\left\|\boldsymbol{H}\left(t_{n}\right)-\boldsymbol{H}^{n}\right\|_{h}^{2}+\epsilon\left\|\boldsymbol{E}\left(t_{n}\right)-\boldsymbol{E}^{n}\right\|_{h}^{2}\right)^{\frac{1}{2}} \\
\leqslant & \sqrt{\mu}\left\|\boldsymbol{H}\left(t_{n}\right)-\boldsymbol{H}^{n}\right\|_{h}+\sqrt{\epsilon}\left\|\boldsymbol{E}\left(t_{n}\right)-\boldsymbol{E}^{n}\right\|_{h} \\
\leqslant & \sqrt{\mu}\left\|\boldsymbol{H}\left(t_{n}\right)-P_{N-2} \boldsymbol{H}\left(t_{n}\right)\right\|_{h}+\left\|\sqrt{\mu} \boldsymbol{E}_{1}{ }^{n}\right\|_{h} \\
& +\sqrt{\epsilon}\left\|\boldsymbol{E}\left(t_{n}\right)-P_{N-2} \boldsymbol{E}\left(t_{n}\right)\right\|_{h}+\left\|\sqrt{\epsilon} \boldsymbol{E}_{2}{ }^{n}\right\|_{h} \\
\leqslant & C T e^{-\bar{\sigma} t_{n}}\left(\tau^{2}+N^{-r}\right) .
\end{aligned}
$$

The proof is completed. 
Remark 3.1. It is remarked that when $\bar{\sigma}=0$, the optimal error estimate in discrete $L^{2}$-norm is established for the energy-conserved method of the Maxwell's equation (2.1).

\section{Numerical experiments}

In this section, we will investigate the numerical behavior of the proposed scheme (2.24)(2.25) presented in Section 2. The rate of convergence and the five discrete conformal energy conservation laws are investigated in detail. All diagrams presented below refer to the numerical integration of (2.3) with $\mu=\epsilon=1$. In addition, the fast solver presented in Ref. [22] is employed to solve the linear equations of (2.24)-(2.25) efficiently.

In order to evaluate the numerical errors, for a fixed $n$, the following formulas are used

$$
L^{\infty}=\max \left\{\mu \max _{j, k, m}\left|\boldsymbol{H}\left(t_{n}\right)-\boldsymbol{H}^{n}\right|, \epsilon \underset{j, k, m}{\max }\left|\boldsymbol{E}\left(t_{n}\right)-\boldsymbol{E}^{n}\right|\right\}
$$

and

$$
L^{2}=\left(\mu\left\|\boldsymbol{H}\left(t_{n}\right)-\boldsymbol{H}^{n}\right\|_{h}^{2}+\epsilon\left\|\boldsymbol{E}\left(t_{n}\right)-\boldsymbol{E}^{n}\right\|_{h}^{2}\right)^{\frac{1}{2}} .
$$

The convergence rate, for a fixed $n$, is defined as

$$
\text { Rate }=\frac{\ln \left(\text { error }_{1} / \text { error }_{2}\right)}{\ln \left(\tau_{1} / \tau_{2}\right)},
$$

where $\tau_{l}$,error $l_{1},\left(l_{1}=1,2\right)$ are step sizes and errors with the step size $\tau_{l_{1}}$, respectively.

Further, for a fixed $n$, errors on the total conformal energies are calculated by using formulas

$$
E r_{l_{2}}^{n}=\left|\mathcal{E}_{l_{2}}^{n}-e^{-2 \bar{\sigma} n \tau} \mathcal{E}_{l_{2}}^{0}\right|, \quad l_{2}=1,2,3
$$

and

$$
\operatorname{Er}_{\widetilde{l}_{w}}^{n}=\left|\mathcal{E}_{\widetilde{l}_{w}}^{n}-e^{-2 \bar{\sigma} n \tau} \mathcal{E}_{\widetilde{l}_{w}}^{0}\right|, \quad \widetilde{l}=4,5 .
$$

\subsection{D Maxwell's equations with the PML}

Firstly, we will focus on the rate of convergence and the conformal energy conservation laws of the 2D Maxwell's equations with the PML. Furthermore, some comparisons between the conformal Fourier pseudo-spectral scheme (CFPS) and the Birkhoffian multisymplectic scheme (BMSS) [32] are displayed. The 2D Maxwell's equations with the 
PML for transverse-electric (TE) waves are

$$
\begin{aligned}
& \frac{\partial E_{x}}{\partial t}=\frac{1}{\epsilon} \frac{\partial H_{z}}{\partial y}-\bar{\sigma} E_{x} \\
& \frac{\partial E_{y}}{\partial t}=-\frac{1}{\epsilon} \frac{\partial H_{z}}{\partial x}-\bar{\sigma} E_{y} \\
& \frac{\partial H_{z}}{\partial t}=\frac{1}{\mu}\left(\frac{\partial E_{x}}{\partial y}-\frac{\partial E_{y}}{\partial x}\right)-\bar{\sigma} H_{z} .
\end{aligned}
$$

Eqs. (4.6)-(4.8) admit the following analytic solutions $(\mu=\epsilon=1)$

$$
\begin{aligned}
& E_{x}=\frac{\sqrt{2}}{2} e^{-\bar{\sigma} t} \cos (2 \sqrt{2} \pi t) \cos (2 \pi x) \sin (2 \pi y), \\
& E_{y}=-\frac{\sqrt{2}}{2} e^{-\bar{\sigma} t} \cos (2 \sqrt{2} \pi t) \sin (2 \pi x) \cos (2 \pi y), \\
& H_{z}=e^{-\bar{\sigma} t} \sin (2 \sqrt{2} \pi t) \cos (2 \pi x) \cos (2 \pi y) .
\end{aligned}
$$

We set the analytic solutions (4.9)-(4.11) at $t=0$ on the domain $\Omega=[0,1] \times[0,1]$ as initial conditions. Table 1 shows the temporal errors and the convergence rates of the different schemes with $\bar{\sigma}=0.1$ and $N_{x}=N_{y}=1024$ at $T=1$. The spatial errors and the convergence rates are investigated in Table 2. As illustrated in Table 1, all of the methods are second order of convergence rate in time, which confirms the theoretical analysis. From Table 2, it is easy to see that the spatial error of the CFPS is very small and almost negligible, and the error is dominated by the time discretization error. It confirms that, for sufficiently smooth problems, the Fourier pseudo-spectral method is of arbitrary order of accuracy. However, the BMS scheme admits relatively large error. The CPU times of different schemes at different spatial steps with $\tau=10^{-6}, \bar{\sigma}=0.1$ and $T=1$ are showed in Table 3. It is clear to see that the CFPS is more efficient than the BMSS. In Fig. 1, we investigate the errors on the conformal energy conservation laws of the CFP and BMS

Table 1: The temporal errors and convergence rates of different schemes with $\bar{\sigma}=0.1$ and $N_{x}=N_{y}=1024$ at $T=1$.

\begin{tabular}{||llllll||}
\hline Scheme & $\tau$ & $L^{2}$ & Rate & $L^{\infty}$ & Rate \\
\hline CFPS & 0.02 & $1.0530 \mathrm{e}-02$ & - & $1.7947 \mathrm{e}-02$ & - \\
& 0.01 & $2.6420 \mathrm{e}-03$ & 1.9949 & $4.5268 \mathrm{e}-03$ & 1.9872 \\
& 0.005 & $6.6108 \mathrm{e}-04$ & 1.9987 & $1.1342 \mathrm{e}-03$ & 1.9968 \\
& 0.0025 & $1.6531 \mathrm{e}-04$ & 1.9997 & $2.8371 \mathrm{e}-04$ & 1.9992 \\
BMSS [32] & 0.02 & $1.0518 \mathrm{e}-02$ & - & $1.7926 \mathrm{e}-02$ & - \\
& 0.01 & $2.6294 \mathrm{e}-03$ & 2.0000 & $4.5053 \mathrm{e}-03$ & 1.9923 \\
& 0.005 & $6.4848 \mathrm{e}-04$ & 2.0196 & $1.1126 \mathrm{e}-03$ & 2.0177 \\
& 0.0025 & $1.5270 \mathrm{e}-04$ & 2.0864 & $2.6207 \mathrm{e}-04$ & 2.0859 \\
\hline
\end{tabular}


Table 2: The spatial errors and convergence rates of different schemes at different spatial steps with $\bar{\sigma}=0.1$ and $\tau=10^{-6}$ and $T=1$.

\begin{tabular}{||llllll||}
\hline Scheme & $N_{x} \times N_{y}$ & $L^{2}$ & Rate & $L^{\infty}$ & Rate \\
\hline CFPS & $16 \times 16$ & $1.2478 \mathrm{e}-010$ & - & $1.6971 \mathrm{e}-010$ & - \\
& $32 \times 32$ & $1.1263 \mathrm{e}-010$ & - & $1.5875 \mathrm{e}-010$ & - \\
& $64 \times 64$ & $1.3661 \mathrm{e}-010$ & - & $1.8197 \mathrm{e}-010$ & - \\
BMSS [32] & $16 \times 16$ & $5.2442 \mathrm{e}-02$ & - & $9.2982 \mathrm{e}-02$ & - \\
& $32 \times 32$ & $1.2965 \mathrm{e}-02$ & 2.0161 & $2.2442 \mathrm{e}-02$ & 2.0507 \\
& $64 \times 64$ & $3.2320 \mathrm{e}-03$ & 2.0041 & $5.5593 \mathrm{e}-03$ & 2.0132 \\
\hline
\end{tabular}

Table 3: The CPU times of different schemes with $\bar{\sigma}=0.1$ and $\tau=10^{-6}$ at $T=1$.

\begin{tabular}{||lll||}
\hline$N_{x} \times N_{y}$ & CFPS & BMSS [32] \\
\hline $8 \times 8$ & $143.9 \mathrm{~s}$ & $154.7 \mathrm{~s}$ \\
$16 \times 16$ & $377.9 \mathrm{~s}$ & $414.4 \mathrm{~s}$ \\
$32 \times 32$ & $1343.9 \mathrm{~s}$ & $1447.2 \mathrm{~s}$ \\
\hline
\end{tabular}

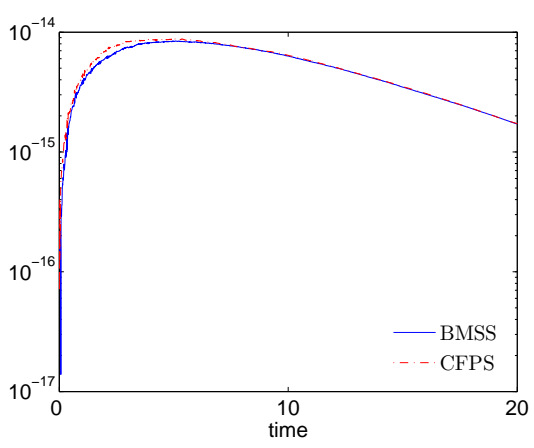

(a)

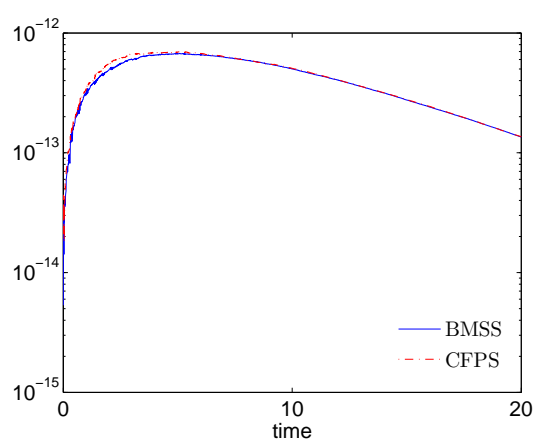

(c)

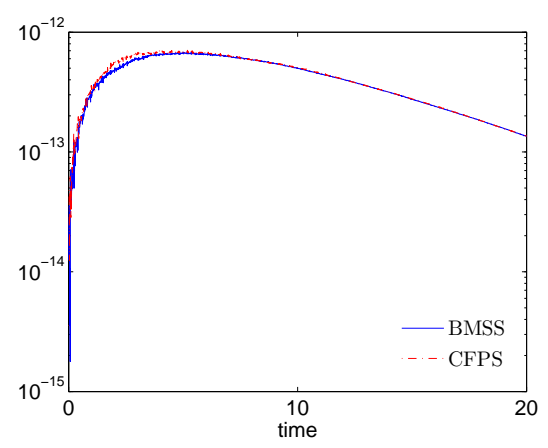

(b)

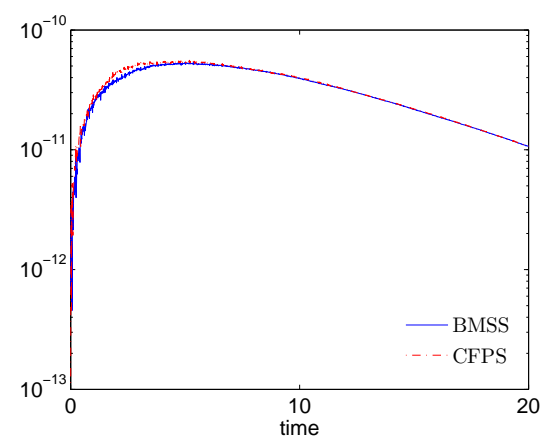

(d)

Figure 1: The errors on the conformal energy conservation laws over the time interval $t \in[0,20]$ with $\bar{\sigma}=0.1$, $N_{x}=N_{y}=128$ and $\tau=0.01$ : (a) $E r_{2}$, (b) $E r_{3}$, (c) $E r_{4_{x}}$, (d) $E r_{5_{x}}$. 
schemes with $N_{x}=N_{y}=128, \tau=0.01$ and $\bar{\sigma}=0.1$. Numerical results show that the CFP and BMS schemes can preserve the conformal energies exactly. Since $E r_{4_{y}}$ and $E r_{5_{y}}$ are close to $E r_{4_{x}}$ and $E r_{5_{x}}$, respectively, the values of $E r_{4_{y}}$ and $E r_{5_{y}}$ are not displayed. Moreover, it has shown that both the popular exponential FDTD Yee's scheme [2] and the traditional multi-symplectic scheme can only approximately preserve the conformal energy conservation laws [32], which implies that the CFP scheme provides distinct advantages in preserving the conformal conservation laws than the exponential FDTD Yee's scheme and the multi-symplectic scheme.

\subsection{D Maxwell's equations with the PML}

Then, the proposed scheme (2.24)-(2.25) is employed to solve the 3D Maxwell's equations with the PML. The 3D Maxwell's equations with the PML admit the analytic solutions, as follows:

$$
\begin{aligned}
& E_{x}=\frac{k_{y}-k_{z}}{\epsilon \sqrt{\mu} w} e^{-\bar{\sigma} t} \cos (w \pi t) \cos \left(k_{x} \pi x\right) \sin \left(k_{y} \pi y\right) \sin \left(k_{z} \pi z\right), \\
& H_{x}=e^{-\bar{\sigma} t} \sin (w \pi t) \sin \left(k_{x} \pi x\right) \cos \left(k_{y} \pi y\right) \cos \left(k_{z} \pi z\right), \\
& E_{y}=\frac{k_{z}-k_{x}}{\epsilon \sqrt{\mu} w} e^{-\bar{\sigma} t} \cos (w \pi t) \sin \left(k_{x} \pi x\right) \cos \left(k_{y} \pi y\right) \sin \left(k_{z} \pi z\right), \\
& H_{y}=e^{-\bar{\sigma} t} \sin (w \pi t) \cos \left(k_{x} \pi x\right) \sin \left(k_{y} \pi y\right) \cos \left(k_{z} \pi z\right), \\
& E_{z}=\frac{k_{x}-k_{y}}{\epsilon \sqrt{\mu} w} e^{-\bar{\sigma} t} \cos (w \pi t) \sin \left(k_{x} \pi x\right) \sin \left(k_{y} \pi y\right) \cos \left(k_{z} \pi z\right), \\
& H_{z}=e^{-\bar{\sigma} t} \sin (w \pi t) \cos \left(k_{x} \pi x\right) \cos \left(k_{y} \pi y\right) \sin \left(k_{z} \pi z\right),
\end{aligned}
$$

where $k_{x}=1, k_{y}=2, k_{z}=-3, \mu=\epsilon=1$, and $w=\left(k_{x}^{2}+k_{y}^{2}+k_{z}^{2}\right) /(\epsilon \mu)$.

We set the analytic solutions (4.12)-(4.17) at $t=0$ on the domain $\Omega=[0,2] \times[0,2] \times[0,2]$ as initial conditions. Table 4 shows the temporal error and convergence rate of the CFP scheme with $\bar{\sigma}=0.1$ and $N_{x}=N_{y}=N_{z}=64$ at $T=1$. It is clear that the CFP scheme is second order of convergence rate in time. The spatial error of the CFP scheme with $\tau=10^{-5}$ at $T=1$ are displayed in Table 5. We find that, similarly to the Section 4.1 , the spatial error is very small and dominated by the time discretization error. It verifies again the fact that,

Table 4: The temporal error and convergence order of the CFP scheme with $\bar{\sigma}=0.1$ and $N_{x}=N_{y}=N_{z}=64$ at $T=1$.

\begin{tabular}{||lllll||}
\hline$\tau$ & $L^{2}$ & Rate & $L^{\infty}$ & Rate \\
\hline 0.01 & $2.1168 \mathrm{e}-02$ & - & $1.1923 \mathrm{e}-02$ & - \\
0.005 & $5.3004 \mathrm{e}-03$ & 1.9978 & $2.9711 \mathrm{e}-03$ & 2.0046 \\
0.0025 & $1.3256 \mathrm{e}-03$ & 1.9994 & $7.4218 \mathrm{e}-04$ & 2.0012 \\
0.00125 & $3.3143 \mathrm{e}-04$ & 1.9999 & $1.8551 \mathrm{e}-04$ & 2.0002 \\
\hline
\end{tabular}


Table 5: The spatial error of the CFP scheme with $\bar{\sigma}=0.1$ and $\tau=10^{-5}$ at $T=1$.

\begin{tabular}{||lll||}
\hline$N_{x} \times N_{y} \times N_{z}$ & $L^{2}$ & $L^{\infty}$ \\
\hline $8 \times 8 \times 8$ & $2.1212 \mathrm{e}-08$ & $1.1838 \mathrm{e}-08$ \\
$16 \times 16 \times 16$ & $2.1212 \mathrm{e}-08$ & $1.1841 \mathrm{e}-08$ \\
$32 \times 32 \times 32$ & $2.1212 \mathrm{e}-08$ & $1.1844 \mathrm{e}-08$ \\
\hline
\end{tabular}

for the sufficiently smooth problem, the CFP scheme is of arbitrary order of accuracy in space. The errors on the five discrete conformal energies over the time interval $t \in[0,20]$ with $\bar{\sigma}=0.1, N_{x}=N_{y}=N_{z}=32$ and $\tau=0.01$ are presented in Fig. 2. It is obvious that the conformal energy conservation laws can be preserved exactly by our scheme.

\section{Concluding remarks}

In this paper, we have developed a novel conformal Fourier pseudo-spectral (CFP) method for the Maxwell's equations with the PML. We show that the CFP scheme is unconditionally stable and preserves the discrete conformal symplectic conservation law as well as the five discrete conformal energy conservative laws. Furthermore, with the aid of the energy method, we establish the optimal error estimate of the CFP scheme at the order of $\mathcal{O}\left(\tau^{2}+N^{-r}\right)$ in discrete $L^{2}$-norm, where $\tau$ is the time step and $N$ is the collocation points used in the spectral method. Numerical results verify the theoretical analysis. In addition, compared with the Birkhoffian multi-symplectic scheme, our scheme is more efficient and provides smaller error in space. Due to the fact that the system of the Maxwell's equations with the PML is not a Hamiltonian system with a constant structure matrix, the sixth order energy-conserved method presented in Ref. [22] cannot be generalized directly to the Maxwell's equations with the PML. Therefore, developing a conformal energy-conserved scheme with a sixth order accuracy for the Maxwell's equations with the PML will be the subject of our future research.

\section{Acknowledgments}

The authors would like to express sincere gratitude to the reviewers for their constructive suggestions which help to improve the quality of this paper. This work is supported by the National Natural Science Foundation of China (Grant Nos. 11771213, 41504078), the National Key Research and Development Project of China (Grant No. 2016YFC0600310). The research is supported by National Key R\&D Program of the Ministry of Science and Technology of China with the Project "Integration Platform Construction for Joint Inversion and Interpretation of Integrated Geophysics" (Grant No. 2018YFC0603500), the Major Projects of Natural Sciences of University in Jiangsu Province of China (Grant No. 15KJA110002) and the Priority Academic Program Development of Jiangsu Higher Education Institutions. 


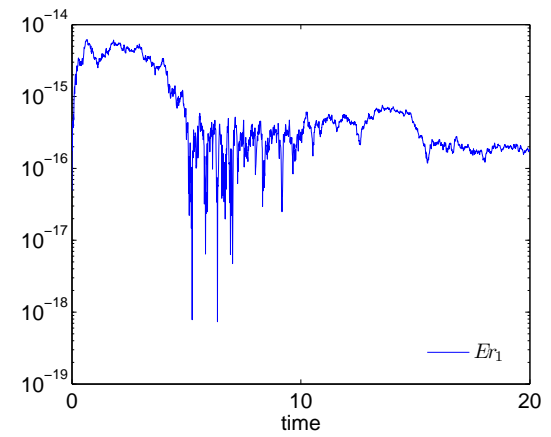

(a)

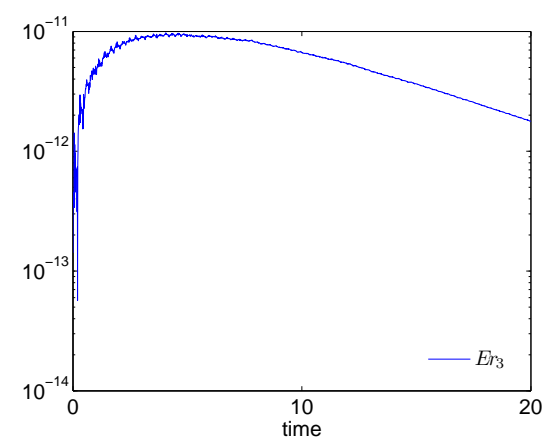

(c)

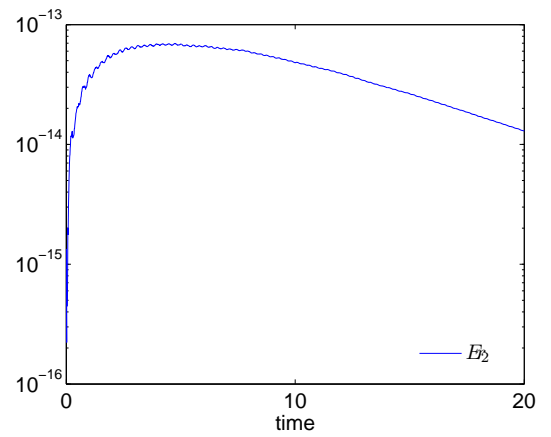

(b)

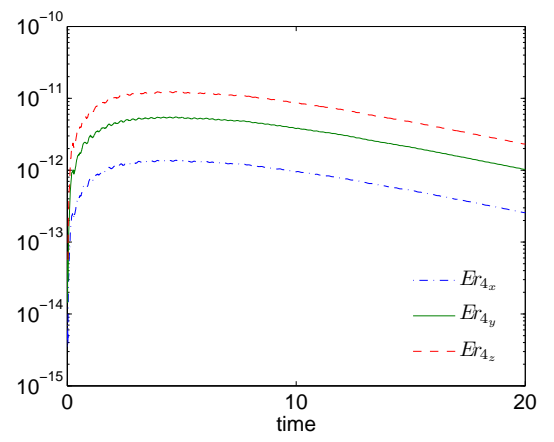

(d)

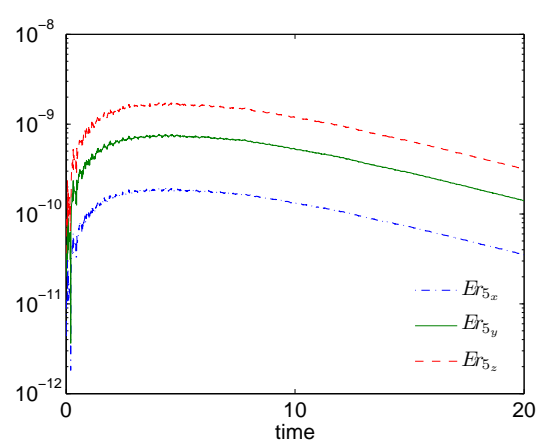

(e)

Figure 2: The errors on the conformal energy conservation laws over the time interval $t \in[0,20]$ with $\bar{\sigma}=0.1$, $N_{x}=N_{y}=N_{z}=32$ and $\tau=0.01$.

\section{References}

[1] N. Anderson and A.M. Arthurs. Helicity and variational principles for Maxwell's equations. Int. J. Electron., 54:861-864, 1983.

[2] J.P. Bérenger. A perfectly matched layer for the absorption of electromagnetic waves. J. Comput. Phys., 114:185-200, 1994. 
[3] J.P. Bérenger. Three-dimensional perfectly matched layer for the absorption of electromagnetic waves. J. Comput. Phys., 127:363-379, 1996.

[4] A. Bhatt, D. Floyd, and B.E. Moore. Second order conformal symplectic schemes for damped Hamiltonian systems. J. Sci. Comput., 66:1234-1259, 2016.

[5] T.J. Bridges and S. Reich. Numerical methods for Hamiltonian PDEs. J. Phys A: Math. Gen., 39:5287-5320, 2006.

[6] J.X. Cai, J.L. Hong, Y.S. Wang, and Y.Z. Gong. Two energy-conserved splitting methods for three-dimensional time-domain Maxwell's equations and the convergence analysis. SIAM. J. Numer. Anal., 53:1918-1940, 2015.

[7] J.X. Cai, Y.S. Wang, and Y.Z. Gong. Numerical analysis of AVF methods for threedimensional time-domain Maxwell's equations. J. Sci. Comput., 66:141-176, 2016.

[8] W.J. Cai, Y.S. Wang, and Y.Z. Song. Numerical dispersion analysis of a multi-symplectic scheme for the three dimensional Maxwell's equations. J. Comput. Phys., 234:330-352, 2013.

[9] C. Canuto and A. Quarteroni. Approximation results for orthogonal polynomials in Sobolev spaces. Math. Comput., 38:67-86, 1982.

[10] J.B. Chen and M.Z. Qin. Multi-symplectic Fourier pseudospectral method for the nonlinear Schrödinger equation. Electr. Trans. Numer. Anal., 12:193-204, 2001.

[11] W.B. Chen, X.J. Li, and D. Liang. Energy-conserved splitting FDTD methods for Maxwell's equations. Numer. Math., 108:445-485, 2008.

[12] W.B. Chen, X.J. Li, and D. Liang. Energy-conserved splitting finite-difference time-domain methods for Maxwell's equations in three dimensions. SIAM. J. Numer. Anal., 48:1530-1554, 2010.

[13] K. Feng and M.Z. Qin. Symplectic geometric algorithms for Hamiltonian systems. Springer and Zhejiang Science and Technology Publishing House, Heidelberg Hangzhou, 2010.

[14] H. Fu, W.E. Zhou, X. Qian, S.H. Song, and L.Y. Zhang. Conformal structure-preserving method for damped nonlinear Schrödinger equation. Chin. Phys. B., 25:110201, 2016.

[15] Y.Z. Gong, J.X. Cai, and Y.S. Wang. Some new structure-preserving algorithms for general multi-symplectic formulations of Hamiltonian PDEs. J. Comput. Phys, 279:80-102, 2014.

[16] Y.Z. Gong, Q. Wang, Y.S. Wang, and J.X. Cai. A conservative Fourier pseudo-spectral method for the nonlinear Schrödinger equation. J. Comput. Phys., 328:354-370, 2017.

[17] E. Hairer, C. Lubich, and G. Wanner. Geometric Numerical Integration: Structure-Preserving Algorithms for Ordinary Differential Equations. Springer-Verlag, Berlin, 2nd edition, 2006.

[18] T. Hirono, W. Lui, S. Seki, and Y. Yoshikuni. A three-dimensional fourth-order finitedifference time-domain scheme using a symplectic integrator propagator. IEEE Trans. Microwave Theory Tech., 49:1640-1648, 2001.

[19] J.L. Hong and L.H. Ji. Energy evolution of multi-symplectic methods for Maxwell equations with perfectly matched layer boundary. J. Math. Anal. Appl., 439:256-270, 2016.

[20] J.L. Hong, L.H. Ji, and L.H. Kong. Energy-dissipation splitting finite-difference time-domain method for Maxwell equations with perfectly matched layers. J. Comput. Phys., 269:201-214, 2014.

[21] J.D. Jackson. Classical electrodynamics. John Wiley \& Sons Inc., New York, third ed edition, 1998.

[22] C.L. Jiang, W.J. Cai, Y.S. Wang, and H.C. Li. A novel sixth order energy-conserved method for three-dimensional time-domain Maxwell's equations. arXiv preprint, arXiv:1705.08125, 2017.

[23] L.H. Kong, J.L. Hong, and J.J. Zhang. Splitting multisymplectic integrators for Maxwells equations. J. Comput. Phys., 229:4259-4278, 2010. 
[24] X.L. Kong, H.B. Wu, and F.X. Mei. Structure-preserving algorithms for Birkhoffian systems. J. Geom. Phys., 62:1157-1166, 2012.

[25] Q.H. Liu. The PSTD algorithm: a time-domain method requiring only two cells per wavelength. Microw. Opt. Technol. Lett., 15:158-165, 1997.

[26] C. Lubich. On splitting methods for Schröinger-Poisson and cubic nonlinear Schrödinger equations. Math. Comput., 77:2141-2153, 2008.

[27] R.I. McLachlan and M. Perlmutter. Conformal Hamiltonian systems. J. Geom. Phys., 39:276300, 2001.

[28] R.I. McLachlan and G.R.W. Quispel. Splitting methods. Acta Numer., 11:341-434, 2002.

[29] B.E. Moore, L. Noreña, and C.M. Schober. Conformal conservation laws and geometric integration for damped Hamiltonian PDEs. J. Comput. Phys., 232:214-233, 2013.

[30] J. Shen and T. Tang. Spectral and High-Order Methods with Applications. Science Press, Beijing, 2006.

[31] G. Strang. On the construction and comparison of difference schemes. SIAM J. Numer. Anal., 5:506-517, 1968.

[32] H.L. Su and S.T. Li. Energy/dissipation-preserving Birkhoffian multi-symplectic methods for Maxwell's equations with dissipation terms. J. Comput. Phys., 311:213-240, 2016.

[33] H.L. Su, M.Z. Qin, and R. Scherer. A multisymplectic geometry and a multisymplectic scheme for Maxwell's equations. Int. J. Pure. Appl. Math., 34:1-17, 2007.

[34] H.L. Su, M.Z. Qin, Y.S. Wang, and R. Scherer. Multi-symplectic Birkhoffian structure for PDEs with dissipation terms. Phys. Lett. A, 374:2410-2416, 2010.

[35] Y.J. Sun and Z.J. Shang. Structure-preserving algorithms for Birkhoffian systems. Phys. Lett. A, 336:358-369, 2005.

[36] Y.J. Sun and P.S.P. Tse. Symplectic and multi-symplectic numerical methods for Maxwell's equations. J. Comput. Phys., 230:2076-2094, 2011.

[37] Y.S. Wang and J.L. Hong. Multi-symplectic algorithms for Hamiltonian partial differential equations. Commun. Appl. Math. Comput., 27:164-230, 2013.

[38] K.S. Yee. Numerical solution of initial boundary value problems involving Maxwell's equations in isotropic media. IEEE Trans. Antennas Propag., 14:302-307, 1966.

[39] H.J. Zhu, S.H. Song, and Y.M. Chen. Multi-symplectic wavelet collocation method for Maxwell's equations. Adv. Appl. Math. Mech., 3:663-688, 2011. 